\title{
Anemarrhenae Rhizoma: Material Basis and Mechanism of Its Renal-Yin-Deficiency Syndrome Treating Effect and Its Effect Enhancement after Salt Stir-frying
}

\section{Shu Han}

Beijing University of Chinese Medicine https://orcid.org/0000-0001-5223-2653

\section{Jinkai Xu}

Beijing University of Chinese Medicine

Weifei Li

China Beijing Tong Ren Tang

Xiaoyan Lu

Beijing University of Chinese Medicine

Meiru Zhi

Beijing University of Chinese Medicine

Kaiyang Liu

Beijing University of Chinese Medicine

Xitao Han

Beijing University of Chinese Medicine

Ya'nan Tang

Beijing University of Chinese Medicine

\section{Ziqin Liu}

Beijing University of Chinese Medicine

Hongyue Wang

Beijing University of Chinese Medicine

\section{Zhigang Yang}

School of Pharmacy Lanzhou University

Fei Li

Beijing University of Chinese Medicine

Hong Du ( $\square$ duhong@vip.163.com )

\section{Research}

Keywords: Anemarrhenae Rhizoma, TCM Herb Processing, Salt Stir-frying, Renal Yin Deficiency Syndrome, Mangiferin, Content Determination 
Posted Date: July 23rd, 2020

DOI: https://doi.org/10.21203/rs.3.rs-45311/v1

License: (c) (i) This work is licensed under a Creative Commons Attribution 4.0 International License. Read Full License 


\section{Abstract}

Background This study aims to find out renal-yin-deficiency syndrome (RYDS) treating component of Anemarrhenae Rhizoma (AR) and its RYDS-treating mechanism, the contents of neomangiferin and mangiferin in raw AR after salt stir-frying, and the cause of enhancement of RYDS-treating effect of salt stir-fried AR.

Methods HPLC method was used for determining contents of mangiferin and neomangiferin in the various AR samples and simulative salt stir-fried neomangiferin. Western Blot, ELISA, RT-PCR and some conventional methods were used to investigate the RYDS-treating effect of raw AR, salt stir-fried AR and mangiferin.

Results Content of neomangiferin decreased and that of mangiferin increased in water stir-fried AR and salt stir-fried AR; HPLC chromatogram showed a mangiferin peak in simulative salt stir-fried neomangiferin; both AR samples and mangiferin exerted RYDS-treating effect by reducing/increasing certain general and specific physiological indexes, reducing levels of T3, T4, FT3, FT4, CAMP, and the ratio of cAMP/cGMP; elevating levels of TSH and cGMP; and increasing mRNA and protein expressions of $\mathrm{PI3K} / \mathrm{AKT} / \mathrm{mTOR}$ pathway in kidney in RYDS rats. The RYDS-treating effect of salt stir-fried AR and mangiferin was stronger than that of raw AR.

Conclusions Results of the study show that mangiferin in AR is the material basis of the RYDS-treating effect, the mechanism of the effect is rectifying and adjusting a series of physiological, endocrinoimmunological and genetic abnormalities caused by RYDS, and enhancement of effect of salt stir-fried $A R$ is because of the increase in the content of mangiferin.

\section{Background}

As an important herb used in traditional Chinese medicine (TCM), Anemarrhenae Rhizoma (AR) comes from the dried rhizome of Anemarrhena asphodeloides Bge. [1]. According to the recordings in Shennong's Classic of Materia Medica, a first-appeared book on traditional Chinese pharmacy (first published about in 220-265 AD), AR was first mentioned by Erhya (an earliest Chinese encyclopedia first published about in 475-221 BC) and has been used as a herb for more than 2000 years in China. Shennong's Classic of Materia Medica holds that AR is medicinally characterized by a bitter/sweet flavor and a cold/cool nature, exerting the effects of clearing out heat/fire and nourishing yin and moistening dryness [2], and in TCM practice, AR is considered to be able to clear out fire from the lung and treat renal dryness and heat. AR contains complex categories of chemical components, mainly including steroidal saponins, phenylpropanoids, alkaloids and flavonoids, and among of them, the anemarrhena saponins and mangiferin are principal biologically active components according to the existing literature $[3,4]$. Anemarrhena saponins has been found to have senile dementia-preventing or -curing[5,6], cerebral ischemia injury-protecting[7], and blood pressure-lowering effects[8, 9], etc., and mangiferin has been found to have anti-inflammatory, anti-tumor, immunomodulatory and renal function-protecting effects[10, 
11, 12]. In TCM clinical practice, AR is used in both raw and processed forms. The methods for processing AR mainly include the grain-liquor stir-frying, bran stir-frying and salt stir-frying. The salt stirfried AR decoction slices were first recorded in the TCM classic Bianque Xinshu (first published about in 1146)[13]. In the Pharmacopoeia of the People's Republic of China (2015-edition), both raw AR decoction slices and salt stir-fried AR decoction slices were included. According to the TCM herb processing theories, AR, after being stir-fried in salt, can be specially guided to the Kidney Channel of Foot-Shaoyin and exert a stronger effect for nourishing renal yin. The salt stir-fried AR, in TCM clinical practice, is frequently used for treating renal yin deficiency syndrome (RYDS). For example, salt stir-fried AR is just one of the ingredients of a TCM patent medicine Zhibai Dihuang Pills, which was recorded in the TCM classic Yizong Jinjian (first published in about 1742 AD)[14], for treating renal yin deficiency, tidal fever and night sweat, and spermatorrhea. Results of modern medical researches suggest that salt stir-fried AR can be used to treat some diseases caused by RYDS, such as diabetes, hypertension and nephritis[15, 16 , $17,18,19]$. Some TCM herb researchers have found in their pharmaceutical and pharmacological researches that the content of neomangiferin and anemarrhena saponins can be decreased and that of mangiferin can be increased in AR after being stir-fried in salt[20, 21, 22, 23], and they have also found that the RYDS-treating effect of the salt stir-fried AR can be enhanced to a certain extent[24, 25], compared with that of the raw AR. Up to now, however, the material basis and the mechanism of the RYDS-treating effect of $A R$, the cause of the change in the contents of neomangiferin and mangiferin in the AR after being stir-fried in salt, and the cause of the enhancement of the RYDS-treating effect of the salt stir-fried $A R$, all remain unclear.

In the present research, we carried out a series of HPLC determinations and pharmacological experiments to investigate the principal chemical components and the RYDS-treating effect of both raw AR and salt stir-fried AR, in order to solve the above-mentioned problems.

\section{Materials And Methods}

\section{Experimental Animals}

56 SPF male SD rats weighing 250-280 g were purchased from Beijing Vital River Laboratory Animal Technology Co., Ltd. (Beijing, China), and the license number of experimental animals was SCXK $(\mathrm{J})$ 2016-0002. The rats were raised in the animal feeding room of Beijing University of Chinese Medicine (BUCM) under specified conditions, i.e. temperature: $22 \pm 2{ }^{\circ} \mathrm{C}$; relative humidity: $50 \pm 5 \%$; and 12/12 $\mathrm{h}$ light-dark cycle. All the experimental rats were audited and approved by the Animal Experimental Welfare Ethics Committee of BUCM.

\section{Instruments and Equipment}

(1) Analytical Balance (Model FA1104B), purchased from Shanghai Yueping Scientific Instrument Co.,Ltd. (Shanghai, China); (2) Ultrasonic Cleaner (Model SB25-12DTDN), purchased from Ningbo Xinzhi Biotechnology Co.,Ltd. (Ningbo, China); (3) High-Speed Universal Shredder (Model FW100), purchased from Beijing Kewei Yongxing Instrument Co.,Ltd. (Beijing, China); (4) Electric Thermostatic Drying Oven 
(Model DHG-9070A), purchased from Changzhou Nuoji Instrument Co.,Ltd. (Jiangsu, China); (5) Salt Stirfrying Pot (Model PC32Y4), purchased from Wuhan SUPOR Cooker Co.,Ltd. (Wuhan, China); (6) Electronic Thermometer (Model MC-246), purchased from Omron (China) Co.,Ltd. (Beijing, China); (7) Electronic Balance (Model JY20002), purchased from Shanghai Hengping Instrument Factory (Shanghai, China); (8) High-Speed Refrigerating Cryogenic Centrifuge (Model BY-R20), purchased from Beijing Baiyang Medical Equipment Co.,Ltd. (Beijing, China); (9) Microplate Reader (Model SIN601-0723), purchased from BioGene Technology Limited (Hong Kong, China); (10) Intelligent Hot Plate Instrument (Model YLS-6B), purchased from Beijing Zhongshi Dichuang Science and Technology Development Co.,Ltd. (Beijing, China); (11) Waters 2965 High Performance Liquid Chromatograph (2965 Chromatographic Pump, 2996 Detector, and Empower Workstation), purchased from Waters Technology (Shanghai) Co.,Ltd. (Shanghai, China); (12) SunFire $\mathrm{C}_{18}$ Chromatographic Column $(4.6 \mathrm{~mm} \times 250 \mathrm{~mm}, 5 \mu \mathrm{m})$, purchased from Waters Technology (Shanghai) Co.,Ltd. (Shanghai, China); (13) PCR Amplifier (Model MA-6000), purchased from Suzhou Yarui Biotechnology Co.,Ltd. (Suzhou, China); (14) Electrophoresis Apparatus (Model DYY-TC), purchased from Beijing Liuyi Biotechnology Co.,Ltd. (Beijing, China); (15) Gel Imaging System (Model 5200), purchased in Shanghai Tanon Science \& Technology Co., Ltd. (Shanghai, China). (16) Non-contact Infrared Thermometer (Model: SmartSensor AR882), purchased from Dongguan Wanchuang Electronic Products Co., Ltd. (Dongguan, China); (17) Electric Stove (Model TL1622), purchased from Foshan Aisi Kaiqi Electric Co., Ltd. (Foshan, China); and (18) Salinometer, pH meter (Model 86555, 10150893), purchased from Hengxin Technology Co., Ltd. (Taiwan, China).

\section{Medicines}

(1) Raw AR Decoction Slices: Purchased from Beijing Shuangqiao Yanjing Decoction Slices Factory (Batch Number: 1904083, Beijing, China), and identified as the dried slices of the rhizome of Anemarrhena asphodeloides Bge., a plant of Liliaceae family, by Prof. Jingjuan Wang of the School of Chinese Materia Medica, BUCM, and the voucher specimens were deposited at the same school. In the experiments with rats, the raw AR decoction slices were used in the dosage form of decoction, which was prepared according to the procedure of first taking $100 \mathrm{~g}$ of raw AR decoction slices and putting it into a container, next adding 10 times the amount of water into it for 30 min of soaking, then decocting the soaked raw AR decoction slices for 2 times, 1 hour for each time, and finally, mixing the decoctions and concentrating the mixture to a concentration of $0.75 \mathrm{~g} / \mathrm{mL}$.

(2) Salt Stir-fried AR Decoction Slices: Prepared by ourselves according to the procedure[26] of first taking $100 \mathrm{~g}$ of raw AR decoction slices and put it into a container, then adding $60 \mathrm{~mL} 2 \%$ salt solution into it for a thorough moistening, and finally, stir-frying the moistened decoction slices at $160-170^{\circ} \mathrm{C}$ for $20 \mathrm{~min}$. In the experiments with rats, the salt stir-fried AR decoction slices were used in the dosage form of decoction, which was prepared according to the procedure of first taking $100 \mathrm{~g}$ of the salt stir-fried AR decoction slices and putting it into a container, next adding 10 times the amount of water into it for 30 min of soaking, then decocting the soaked salt stir-fried AR decoction slices for 2 times, 1 hour for each time, and finally, mixing the decoctions and concentrating the mixture to a concentration of $0.75 \mathrm{~g} /$ $\mathrm{mL}$. 
(3) Water Stir-fried AR Decoction Slices: Prepared by ourselves according to the procedure [26] of first taking $100 \mathrm{~g}$ of raw AR decoction slices and putting it into a container, then adding $60 \mathrm{~mL}$ water into it for a thorough moistening, and finally, stir-frying the moistened decoction slices at $160-170^{\circ} \mathrm{C}$ for $20 \mathrm{~min}$. In the HPLC determination of chemical components, a sample solution of the water stir-fried AR decoction slices was prepared. (For details, see 3.2.1.2 of this paper.)

(4) Thyroxine Tablets: Purchased from Shandong Laiyang Biochemical Pharmaceutical Factory (Batch Number: H31022151, Shandong, China). In the experiments with rats, thyroxine tablets were used in the form of a suspension, which was prepared according to the procedure of first taking an appropriate amount of thyroxine tablets and put it into a mortar, next pestling it into powder, then adding $0.5 \% \mathrm{CMC}$ $\mathrm{Na}$ solution (prepared with sterile saline) into the powder, and finally, well mixing the powder of thyroxine tablets and the $0.5 \% \mathrm{CMC}-\mathrm{Na}$ solution for getting a suspension with a concentration of $1.25 \mathrm{mg} / \mathrm{mL}$. The suspension should be kept in a refrigerator at $4^{\circ} \mathrm{C}$ and well shaken before use.

(5) Mangiferin Powder: Purchased from Shanghai Yuanye Bio-Technology Co., Ltd. (Purity $\geq 90 \%$, Batch Number: C21MGY61363, Shanghai, China). In the experiments with rats, mangiferin was used in the form of a suspension, which was prepared according to the procedure of first taking an appropriate amount of mangiferin and put it into a container, then adding $0.5 \% \mathrm{CMC}$-Na solution (prepared with sterile saline) into it, and finally well mixing the mangiferin and the $0.5 \% \mathrm{CMC}-\mathrm{Na}$ solution for getting a suspension with a concentration of $20 \mathrm{mg} / \mathrm{mL}$. The suspension should be kept in a refrigerator at $4{ }^{\circ} \mathrm{C}$ and well shaken before use.

(6) Liuwei Dihuang Pills: Purchased from the Affiliated Pharmaceutical Factory of Beijing Tongrentang Science and Technology Development Co.,Ltd. (Batch Number: Z19993068, Beijing, China). In the experiments with rats, Liuwei Dihuang Pills were used in the form of a suspension, which was prepared according to the procedure of first taking an appropriate amount of Liuwei Dihuang Pills and put it into a container, then adding $0.5 \%$ CMC-Na solution (prepared with sterile saline) into it, and finally well mixing the Liuwei Dihuang Pills and the $0.5 \% \mathrm{CMC}$-Na solution for getting a suspension with a concentration of $0.52 \mathrm{~g} / \mathrm{mL}$. The suspension should be kept in a refrigerator at $4^{\circ} \mathrm{C}$ and well shaken before use.

(7) Propylthiouracil Tablets: Purchased from Shanghai Yuanye Biotechnology Co.,Ltd. (Batch Number: H26J9Z66436, Shanghai, China). In the experiments with rats, propylthiouracil tablets were used in the form of a suspension, which was prepared according to the procedure of first taking an appropriate amount of propylthiouracil tablets and put it into a mortar, next pestling it into powder, then adding $0.5 \%$ CMC-Na solution (prepared with sterile saline) into it, and finally well mixing the powder of propylthiouracil tablets and the $0.5 \% \mathrm{CMC}$-Na solution for getting a suspension with a concentration of $5 \mathrm{mg} / \mathrm{mL}$. The suspension should be kept in a refrigerator at $4^{\circ} \mathrm{C}$ and well shaken before use.

\section{Reference Substances}

(1) Mangiferin (Batch Number: 111607-201503, purity $\geq 98 \%$ ), (2) Neomangiferin (Batch Number: 111839-201504, purity $\geq 98 \%$, and (3) Anemarrhenasaponin B (Batch Number: 111839-201505, purity 
$\geq 98 \%$ ), were purchased from the National Institutes for Food and Drug Control (Beijing, China); (4) Icariin (Batch Number: T09N6B5664, purity $\geq 98 \%$ ) was purchased from Shanghai Yuanye Biotechnology Co., Ltd. (Shanghai, China).

\section{Main Reagents}

(1)Triiodothyronine (T3) Kit (Batch Number: AE16221RA), (2) Tetraiodothyronine (T4) Kit (Batch Number: AE16236RA), (3) Thyroid Stimulating Hormone (TSH) kit (Batch Number:AE13132MO), (4) Free Triiodothyronine (FT3) Kit (Batch Number: AE43217MO), (5) Free Tetraiodothyronine (FT4) Kit (Batch Number: AE43100MO), (6) Cyclic Adenosine Monophosphate (cAMP) Kit (Batch Number: AE61565RA) and (7) Cyclic Guanosine Monophosphate (cGMP) Kit (Batch Number: AE60957M0), were all purchased from Nanjing Jiancheng Bioengineering Institute (Nanjing, China); (8) Real-Time PCR Kit (Model: AQ13101) and (9) TRIZOL Kit (Model: H10318) were all purchased from Beijing TransGen Biotech Co., Ltd. (Beijing, China); (10) BCA Protein Assay Kit (Model: PC0020) and (11) ECL Hypersensitive Luminous Liquid Kit (Model: PE0010A + B), RIPA Lysate (Model: R0020), were all purchased from Beijing Solarbio Technology Co., Ltd. (Beijing, China); (12) GAPDH Internal Reference Antibody (Model: TA-08), (13) Goat Anti-Rat-HRP (Model: 134560), (14) Goat Anti-Rabbit-HRP (Model: ZB-2301) and (15) Rabbit Anti-GoatHRP (Model: ZB-2306) were all purchased from Beijing Zhongshan Jinqiao Biotechnology Co., Ltd. (Beijing, China); (16) Rabbit Anti-MTOR (Model: Ab2732), (17) Rabbit Anti-PI3KP85a (Model: Ab191606), and (18) Rabbit anti-AKT1/2/3 (Model: Ab179463) were purchased from Abcam (Shanghai, China); (19) Glacial Acetic Acid (Chromatographic Grade), (20) Acetic Acid (Chromatographic Grade), (21) Ethanol (Chromatographic Grade) and (22) Methanol (Chromatographic Grade), were all purchased from Thermo Fisher. Scientific (China) Co., Ltd.(Shanghai, China); (23) Ultrapure Water was prepared with Milli-Q System (Milford, USA); and (24) Sterile Saline (Batch Number: 1901013202) was purchased from Hebei Shijiazhuang No.4 Pharmaceutical Co., Ltd. (Hebei, China).

\section{Experimental Methods}

\section{Conditions for Performing HPLC Determinations}

HPLC determinations were performed by use of the Waters HPLC 2965 (with 2695 chromatographic pump, Empower workstation, ultraviolet detector), under the conditions of SunFire $\mathrm{C}_{18}$ column $(4.6 \mathrm{~mm} \times$ $250 \mathrm{~mm}, 5 \mu \mathrm{m}$; column temperature: $30^{\circ} \mathrm{C}$ ); mobile phase: acetonitrile for phase $A$ and $0.2 \%$ glacial acetic acid-water for phase $B$; gradient elution (0-10 min, 15-25\%A; 10-20 min, 25-80\%A; 20-30 min, $80-$ $15 \% \mathrm{~A}$ ); the flow rate: $1.0 \mathrm{~mL} . \mathrm{min}-1$; the wavelength of the detector: $258 \mathrm{~nm}$; and the injecting volume of the sample: $20 \mu \mathrm{L}$.

\section{Preparation of the Sample Solutions of Raw AR, Salt Stir-fried AR and Water Stir-fried AR for a HPLC Determination}

(1) Preparation of the Sample Solutions of Raw AR, Salt Stir-fried AR and Water Stir-fried AR: Accuratelyweighed $0.1 \mathrm{~g}$ powder (through sieve No.3) of raw AR (or salt stir-fried AR, or water stir-fried AR) was put into a conical flask with cover, and accurately-measured $25 \mathrm{~mL}$ of $50 \%$ ethanol was added into the flask. The mixture of the powder and ethanol, after weighing, was undergone an ultrasonic treatment (power: 400W; frequency: $40 \mathrm{kHz}$ ) for 30 minutes, and after cooling, the mixture was replenished with $50 \%$ 
ethanol for its loss of weight. And then, the mixture was filtered after being shaken well, and the subsequent filtrate was taken to filter again through a $0.45 \mu \mathrm{m}$ microfiltration membrane. The final filtrate was kept for later use.

(2) Preparation of the Sample Solutions of Mangiferin, Neomangiferin, Icariin and Anemarrhenasaponin BII: First, an appropriate accurately-weighed amount of mangiferin (or neomangiferin, or icariin) was put into a $25 \mathrm{~mL}$ volumetric flask, and then, an appropriate accurately-measured volume of $50 \%$ ethanol was added into the volumetric flask to gain a mangiferin solution at a concentration of $1.25 \mathrm{mg} / \mathrm{mL}$ (or to gain a neomangiferin solution at a concentration of $1.54 \mathrm{mg} / \mathrm{mL}$, or an icariin solution at the concentration of $1.05 \mathrm{mg} / \mathrm{mL}$ ). Or similarly, first, an appropriate accurately-weighed amount of anemarrhenasaponin Bll was put into a $25 \mathrm{~mL}$ volumetric flask, and then, appropriate accuratelymeasured volume of $30 \%$ acetone was added into the flask to gain an anemarrhenasaponin Bll solution at a concentration of $0.5 \mathrm{mg} / \mathrm{mL}$.

\section{Preparation of the Sample Solutions of Simulative Salt Stir-fried Neomangiferin and Salt Stir-fried Simulative Neomangiferin Decoction Slices for a HPLC Determination}

(1) Preparation of Simulative Salt Stir-fried Neomangiferin: $2 \mathrm{mg}$ of accurately weighed neomangiferin was put into a heat-stabile container, and then accurately measured $5 \mathrm{~mL}$ of $2 \%$ salt solution was added into the container. After the mixture of the neomangiferin and the salt solution in the container being well shaken, the container holding the mixture was put into an oven and baked at $160-170^{\circ} \mathrm{C}$ for $20 \mathrm{~min}$. Finally, the container was taken out for cooling and the mixture inside it was kept for later use.

(2) Preparation of the Sample Solution of Simulative Salt Stir-fried Neomangiferin: An appropriate amount (accurately weighed) of the simulative salt stir-fried neomangiferin was placed into a conical flask with cover, and an appropriate volume (measured accurately) of $50 \%$ ethanol was added into the flask. The mixture of the simulative salt stir-fried neomangiferin and ethanol, after weighing, was undergone an ultrasonic treatment (power: 400W; frequency: $40 \mathrm{kHz}$ ) for 30 minutes, and after cooling, the mixture was replenished with $50 \%$ ethanol for its loss of weight. And then, the mixture was filtered after being shaken well, and the subsequent filtrate was again taken to filter through a $0.45 \mu \mathrm{m}$ microfiltration membrane. The final filtrate was kept for later use.

(3) Preparation of Salt Stir-fried Simulative Neomangiferin Decoction Slices: An appropriate amount of raw AR decoction slices was put into the percolator, and then, certain volume of $50 \%$ ethanol was put into the percolator to start percolating. When the percolate became colorless, the raw AR decoction slices were taken out of the percolator, and the residual ethanol with the slices was completely removed by volatilization. And finally, the slices were put into an oven to be dried at $50^{\circ} \mathrm{C}$ for $2 \mathrm{~h}$. The dried slices were then considered as "blank AR decoction slices." In order to prepare the salt stir-fried simulative neomangiferin decoction slices, first, an appropriate amount of neomangiferin (weighed accurately) was loaded into the "blank AR decoction slices" to simulate the "neomangiferin decoction slices", which were then mixed with an appropriate amount of $2 \%$ salt solution. After being well moistened with the salt solution, the simulative neomangiferin decoction slices were stir-fried in a special pot at $160-170^{\circ} \mathrm{C}$ for 
20 min. Finally, the salt stir-fried simulative neomangiferin decoction slices were taken out of the pot for cooling for later use. (See Fig. 1)

(4) Preparation of the Sample Solution of the Salt Stir-fried Simulative Neomangiferin Decoction Slices: An appropriate amount (weighed accurately) of the powder (through sieve No.3) of the salt stir-fried simulative neomangiferin decoction slices was put into a conical flask with cover, and an appropriate volume (accurately measured) of $50 \%$ ethanol was added into the flask. The mixture of the powder and ethanol, after weighing, was undergone an ultrasonic treatment (power: $400 \mathrm{~W}$; frequency: $40 \mathrm{kHz}$ ) for 30 minutes, and the mixture, after cooling, was replenished with $50 \%$ ethanol for its loss of weight. Then, after being shaken well, the mixture was filtered and the subsequent filtrate was taken to filter again through a $0.45 \mu \mathrm{m}$ microfiltration membrane. The final filtrate was kept for later use.

\section{Grouping of the Experimental Rats}

All the experimental rats were adaptively raised for 1 week before starting the pharmacological experiments, during which, the rats had a free daily access to standard food and distilled water. After the adaptive raising, according to the random number table method, the rats were assigned to the following 7 groups (8 rats in each group) for a 14-day experiment on the RYDS-treating effect of raw AR and salt stirfried AR. At the end of the experiment, the relevant indexes for evaluating the RYDS-treating effect were detected.

(1) Blank Control (BC) Group: During an experiment, the rats were allowed to have a free daily access to standard food and distilled water, and their mental state, physical condition, behavior, and certain physiological indexes were observed and detected regularly. At the end of the experiment, certain endocrino-immunological and genetic indexes were measured and detected. (For details, see 3.2.2.2 of this paper, and the same below.)

(2) RYDS Model Group: During an experiment, besides being allowed to have a free daily access to standard food and distilled water, the rats were intragastrically given thyroid hormone suspension $(1.25 \mathrm{mg} / \mathrm{mL})$ at a dose of $1 \mathrm{~mL} / 100 \mathrm{~g}$ in the morning, and sterile saline at a dose of $1 \mathrm{~mL} / 100 \mathrm{~g}$ in the afternoon. And their mental state, physical condition, behavior, and certain physiological indexes were observed and detected regularly. At the end of the experiment, certain endocrino-immunological and genetic indexes were measured and detected. The dose of thyroid hormone was decided according to the results of our preliminary experiment. (The same below.)

(3) Raw AR Treating (RART) Group: During an experiment, besides being allowed to have a free daily access to standard food and distilled water, the rats were intragastrically given thyroid hormone suspension $(1.25 \mathrm{mg} / \mathrm{mL})$ at a dose of $1 \mathrm{~mL} / 100 \mathrm{~g}$ in the morning, and the decoction of raw AR $(0.75 \mathrm{~g} / \mathrm{mL})$ at a dose of $1 \mathrm{~mL} / 100 \mathrm{~g}$ in the afternoon. And their mental state, physical condition, behavior, and certain physiological indexes were observed and detected regularly. At the end of the 
experiment, certain endocrino-immunological and genetic indexes were measured and detected. The dose of raw AR was decided according to literature[1]and the results of our preliminary experiment.

(4) Salt Stir-fried AR Treating (SSART) Group: During an experiment, besides being allowed to have a free daily access to standard food and distilled water, the rats were intragastrically given thyroid hormone suspension $(1.25 \mathrm{mg} / \mathrm{mL})$ at a dose of $1 \mathrm{~mL} / 100 \mathrm{~g}$ in the morning, and the decoction of salt stir-fried AR $(0.75 \mathrm{~g} / \mathrm{mL})$ at a dose of $1 \mathrm{~mL} / 100 \mathrm{~g}$ in the afternoon. And their mental state, physical condition, behavior, and certain physiological indexes were observed and detected regularly. At the end of the experiment, certain endocrino-immunological and genetic indexes were measured and detected. The dose of salt stir-fried AR was decided according to literature[1] and the results of our preliminary experiment.

(5) Mangiferin Powder Treating (MPT) Group: During an experiment, besides being allowed to have a free daily access to standard food and distilled water, the rats were intragastrically given thyroid hormone suspension $(1.25 \mathrm{mg} / \mathrm{mL})$ at a dose of $1 \mathrm{~mL} / 100 \mathrm{~g}$ in the morning, and the mangiferin suspension $(20 \mathrm{mg} / \mathrm{mL})$ at a dose of $1 \mathrm{~mL} / 100 \mathrm{~g}$ in the afternoon. And their mental state, physical condition, behavior, and certain physiological indexes were observed and detected regularly. At the end of the experiment, certain endocrino-immunological and genetic indexes were measured and detected. The dose of mangiferin was decided according to literature[27, 28]and the results of our preliminary experiment.

(6) Propylthiouracil Tablets Treating (PTT) Group: During an experiment, besides being allowed to have a free daily access to standard food and distilled water, the rats were intragastrically given thyroid hormone suspension $(1.25 \mathrm{mg} / \mathrm{mL})$ at a dose of $1 \mathrm{~mL} / 100 \mathrm{~g}$ in the morning, and the propylthiouracil suspension $(5 \mathrm{mg} / \mathrm{mL})$ at a dose of $1 \mathrm{~mL} / 100 \mathrm{~g}$ in the afternoon. And their mental state, physical condition, behavior, and certain physiological indexes were observed and detected regularly. At the end of the experiment, certain endocrino-immunological indexes were measured and detected. The dose of propylthiouracil was decided according to literature[29] and the results of our preliminary experiment.

(7) Liuwei Dihuang Pills Treating (LDPT) Group: During an experiment, besides allowed to have a free daily access to standard food and distilled water, the rats were intragastrically given thyroid hormone suspension $(1.25 \mathrm{mg} / \mathrm{mL})$ at a dose of $1 \mathrm{~mL} / 100 \mathrm{~g}$ in the morning, and the Liuwei Dihuang Pills suspension $(0.52 \mathrm{mg} / \mathrm{mL})$ at a dose of $1 \mathrm{~mL} / 100 \mathrm{~g}$ in the afternoon. And their mental state, physical condition, behavior, and certain physiological indexes were observed and detected regularly. At the end of the experiment, certain endocrino-immunological indexes were measured and detected. The dose of Liuwei Dihuang Pills was decided according to literature[30,28]and the results of our preliminary experiment.

\section{Detection of the Relevant Indexes for Evaluating RYDS- treating Effect}

(1) Detection of Certain Physiological Indexes: Both at the time of starting the RYDS modeling and at the time of finishing the RYDS modeling, the values of the variation in body weight, anal temperature, pain 
threshold, water-intake, food-intake and urine volume were measured in the rats in each experimental group, respectively.

(2) Detection of the Organ Index of Certain Immune and Endocrine Organs: At the time of finishing the RYDS modeling, the rats, after body weighing, were anesthetized by an intraperitoneal injection of $1 \%$ sodium pentobarbital and then, their kidneys, adrenal glands, thymus and spleen were immediately separated and washed with pre-cooled sterile saline for removing the blood stains. After being cleaned by rubbing with filter paper, these organs were weighed with an analytical balance for getting their respective wet weight to calculate an organ index. The organ index of a relevant organ was calculated by using one of the following 2 formulas:

Organ Index \% = Organ Wet Weight/Body Weight $\times 100 \%$ (for the kidney, or thymus, or spleen)

Adrenal Gland Index \% = Wet Weight of Adrenal Gland /Body Weight $\times 1000 \%$

(3) Detection of Relevant Endocrine-immunological Indexes in Serum/Plasma: First, the rats were anesthetized by an intraperitoneal injection of $1 \%$ sodium pentobarbital, next, their abdominal aortic blood was collected and put into the anticoagulant blood collecting tube containing heparin sodium, then the blood was centrifuged $(4000 \mathrm{r} / \mathrm{min})$ at $4^{\circ} \mathrm{C}$ for $10 \mathrm{~min}$ with a high-speed refrigerated cryogenic centrifuge, and finally, the obtained serum and plasma were stored at $-80^{\circ} \mathrm{C}$ in a refrigerator for later use. The serumal levels of T3, T4, FT3, FT4, TSH, the plasmatic levels of cAMP and cGMP, and the plasmatic ratio of cAMP/cGMP were detected by use of ELISA. (See an ELISA kit for instructions.)

(4) Detection of the Genetic Index Concerning the mRNA Expression of PI3K/AKT/mTOR: $30 \mathrm{mg}$ of rat renal tissue of the rats in each relevant group was used to extract total mRNA by the Trizol method (Invitrogen), then each group of mRNA was reversely transcribed into cDNA by using a reverse transcription kit, and finally the change in the level of target gene mRNA expression was detected by use of RT-PCR method. The primers used were synthesized by Shanghai Jinglai Biotechnology Co., Ltd. (see Table 1).

(5) Detection of the Genetic Index Concerning the Protein Expression of PI3K/AKT/mTOR: Western Blot was used to detect the protein expression of PI3K/AKT/mTOR of the rats in each relevant group. An appropriate amount of lysate was added to an appropriate amount of rat renal tissue for homogenization, and the mixture was kept still on ice for $30 \mathrm{~min}$. And then, the mixture was transferred to a $1.5 \mathrm{~mL}$ centrifuge tube for centrifuging at $12,000 \mathrm{r} / \mathrm{min}$ for $15 \mathrm{~min}$ at $4^{\circ} \mathrm{C}$. The supernatant of the mixture was collected and used for detecting the protein concentration in the rat renal tissue by the BCA method, and the protein concentrations in the renal tissue of the rats in each relevant group were then adjusted to an identical level. Next, the supernatant with the adjusted protein concentration was analyzed by the SDS-PAGE ( $10 \%$ separating gel and $5 \%$ concentrating gel) electrophoresis at a voltage of $80 \mathrm{~V}$, which was increased to $120 \mathrm{~V}$ when the concentrating gel overflowed from the sample, and the electrophoresis was not terminated until the dye (marker) just started to overflow. After the electrophoresis, the protein bands of the supernatant with the adjusted protein concentration were 
transferred, through wet transfer at a voltage of $100 \mathrm{~V}$, to PVDF membranes, which were then sealed in a container at room temperature for $2 \mathrm{~h}$. Afterwards, the primary antibody (diluted at a ratio of 1:1000) and $\beta$-actin were added to the PVDF membranes, which were then incubated at $4^{\circ} \mathrm{C}$ overnight. The PVDF membranes were then washed with TBST for 3 times, 7 min each time. And then, the secondary antibody was added to the PVDF membranes, which were then incubated once more at room temperature for $2 \mathrm{~h}$. Afterwards, the PVDF membranes were washed with TBST for 3 times, 7 min each time. And then, the working liquid, i.e. an even mixture of the liquid $A$ and liquid $B$ (subordinate to the ECL hypersensitive luminous liquid) at a ratio of 1:1, was dropped on the PVDF membranes to react for $1 \mathrm{~min}$, and the redundant luminous liquid was removed by absorbing with filter paper. Next, the PVDF membranes were coated with the preservative film for exposing and imaging with a chemiluminescent imager. Finally, the molecular weight and gray value of the target band of the PVDF membranes were scanned by the gel image processing system, and a comparison between the gray value of the target band and that of the corresponding $\beta$-actin protein was made to gain a ratio for explaining the level of the relative expression of each histone.

\section{Statistical Analysis of the Experimental Results}

SPSS 16.0 (SPSS, Inc., Chicago, IL, USA) statistical software was used to process all the experimental data, which were expressed as mean $( \pm)$ standard deviation; the comparison of the experimental data between the BC group and the RYDS model group was conducted by t-test, and the difference was considered to be statistically significant when $\mathrm{P}<0.05$; and the comparison of experimental data between the RYDS model group and each of the treating groups (including the RART group, SSART group, MPT group, PTT group and the LDPT group) was conducted by using one-way ANOVA, and the difference was considered statistically significant when $\mathrm{P}<0.05$.

\section{Experimental Results}

Results of HPLC Quantitative Determination of the Principal Chemical Components in Raw AR, Salt Stirfried AR and Water Stir-fried AR

The HPLC chromatograms of both raw AR and salt stir-fried AR (see Fig. 2) show 4 chromatographic peaks, representing neomangiferin, mangiferin, icariin and anemarrhenasaponin BII. Although the peaks of both neomangiferin and mangniferin are higher than those of other components in both raw AR and salt stir-fried AR, compared with raw AR, salt stir-fried AR shows a lower peak of neomangiferin and a higher peak of mangniferin, implying that there happened a transformation of neomangiferin to mangniferin in salt stir-fried AR, which causing an increase in the content of mangiferin and a decrease in the content of neomangiferin. However, the HPLC chromatograms show no implication of any new chemical components formed during the neomangiferin-mangniferin transformation.

The above-mentioned chromatographic peaks of mangiferin and neomangiferin were the characteristic peaks of the chromatograms of both raw AR and salt stir-fried AR, which can be quantitatively expressed with their own relative peak areas. The PCA score plot of the relative peak areas of the chromatographic peaks of mangiferin and neomangiferin (see Fig. 4) show that the samples of raw AR and salt stir-fried 
AR are affiliated to 2 different clusters, which are separated by a relatively long distance, indicating that in the HPLC determination, the contents of the principal chemical components, i.e. mangiferin and neomangiferin, were different in the samples of raw AR and salt stir-fried AR.

Moreover, the peaks of mangiferin in the HPLC chromatograms (Fig. 2-3) implies that in the relevant experiment, the magnitude of the amount of mangiferin in a sample of AR followed the sequence that amount in salt stir-fried AR > amount in water stir-fried AR > amount in raw AR. The above-mentioned sequence can be verified by the numerical values, which were calculated by using the relative peak area method of the contents of mangiferin in raw AR, water stir-fried AR and salt stir-fried AR (see Table 2). The results of the HPLC determination of the contents mangiferin and neomangiferin show that there was a neomangiferin-mangiferin transformation during the process of salt stir-frying or water stir-frying of $A R$, it was found that through calculation, the transformation rate of neomangiferin-mangiferin transformation in water stir-fried AR was $9 \%$ and that in salt stir-fried AR was $14 \%$, and the transformation rate can be calculated by using the following formula:

(amount of mangiferin in Processed AR - amount of mangiferin in raw AR) / amount of neomangiferin in raw AR

Notes: In the above formula, processed AR = salt stir-fried AR or water stir-fried AR.

\section{Results of the HPLC Quantitative Determination of the Simulative Salt Stir-fried Neomangiferin}

The chromatograms of the HPLC determination of simulative salt stir-fried neomangiferin (see Fig. 5) show that before being simulatively stir-fried in salt, neomangiferin only presents 1 peak, but after being simulatively stir-fried in salt, neomangiferin presents 2 peaks, the later appeared one of which is that of mangiferin. The results imply that there was also a neomangiferin-mangiferin transformation during the simulative salt stir-frying of neomangiferin. It can be seen from Fig. 6A-B, that raw AR decoction slices show 2 principal chromatographic peaks corresponding to neomangiferin and mangiferin, while after the extraction of the chemical components by percolation, raw AR decoction slices show almost no any chromatographic peaks in the corresponding positions, implying that the raw AR decoction slices were made free from all chemical components by the percolation (see 3.2.1.3 of this paper) and became basically "blank". Figure $6 \mathrm{C}$ indicates that in the relevant determination, neomangiferin and mangiferin were simultaneously detected in the salt stir-fried simulative neomangiferin decoction slices, which were prepared by "loading" neomangiferin into the "blank AR decoction slices" and salt stir-frying, and the determination results also imply that there was a neomangiferin-mangiferin transformation during salt stir-frying of the simulative neomangiferin decoction slices.

\section{Inducement of the Rat RYDS Model}


It can be seen from the data in Fig. 7-8 that in the relevant experiment, after intragastric administration of the suspension of thyroxine tablets for 14 days, compared with those in the BC group, the rats in the RYDS model group showed typical RYDS-related abnormalities in relevant indexes, including a decrease in the variation of body weight and pain threshold $(P<0.01)$; an increase in anal temperature, waterintake, food-intake and urine volume $(P<0.01)$; an increase in organ indexes of the kidney, adrenal gland and spleen $(P<0.01)$; a decrease in the organ index of the thymus $(P<0.01)$; an increase in the levels of T3, T4, FT3 and FT4 $(\mathrm{P}<0.01)$; an increase in both the level of CAMP and the ratio of cAMP/cGMP $(\mathrm{P}<$ $0.01)$; a decrease in the level of TSH $(P<0.01)$; and a decrease in the level of cGMP $(P<0.05)$. The heat map of cluster analysis of the values of the detected physiological and endocrino-immunological indexes (see Fig. 9) shows that the heat map consists of 2 different areas, the blue one of which, represents the decreased values of relevant indexes, and the red one of which, represents the increased values of relevant indexes. According to the heat map, the BC group and RYDS model group can be easily recognized, implying that the inducement of the rat RYDS model was successful.

\section{Results from the Experiments on the RYDS-treating Effect of the Positive Control Agents Liuwei Dihuang Pills and Propylthiouracil Tablets in the Rats with RYDS}

The data of the RYDS-treating effect of the 2 positive control agents, i.e. Liuwei Dihuang Pills and propylthiouracil tablets are arranged in Fig. 7-8, from which, it can be seen that in the relevant experiments, after intragastric administration of the suspension of Liuwei Dihuang Pills or the suspension of propylthiouracil tablets for 14 days, compared with those in the RYDS model group, the rats in the LDPT group and PTT group showed an increase in pain threshold $(P<0.01)$; a decrease in anal temperature, water-intake, food-intake, and urine volume $(P<0.01)$; a decrease in the organ indexes of the kidney, adrenal gland $(P<0.01)$ and spleen $(P<0.05)$; an increase in the organ index of the thymus $(P<$ $0.01)$; a decrease in the levels of T3, T4, FT4, CAMP, and in the ratio of cAMP/cGMP $(P<0.01)$; and an increase in the level of cGMP (Pख0.05). Compared with those in the RYDS model group, the rats in the LDPT group showed an increase in the variation of body weight $(P<0.05)$ and no obvious change in the levels of FT3 and TSH ( $P>0.05)$; while compared with those in the RYDS model group, the rats in the PTT group showed a decrease in the level of FT3, an increase in the level of TSH $(P<0.01)$, and no obvious variation in body weight $(P>0.05)$. The above-mentioned experimental data explain that in the relevant experiment, Liuwei Dihuang Pills and propylthiouracil tablets exerted their RYDS-treating effect by rectifying and adjusting a series of physiological and endocrino-immunological abnormalities caused by RYDS. In the present research, we used the data of the RYDS-treating effect of the 2 positive agents for reference in the evaluation of the RYDS-treating effect of the experimental medicines included in the present research.

Results from the Experiments on the RYDS-treating Effect of Raw AR, Salt Stir-fried AR and Mangiferin in the Rats with RYDS 
The data of the RYDS-treating effect of the decoctions of raw AR and salt stir-fried AR in the rats with RYDS are arranged in Fig. 10-13. It can be seen that in the relevant experiments after 14 days of intragastric administration of the decoction of raw AR or the decoctions of salt stir-fried AR, compared with those in the RYDS model group, the rats in both the RART group and the SSART group showed an increase in the variation of body weight and pain threshold $(P<0.01)$; a decrease in anal temperature, water-intake, food-intake and urine volume $(P<0.01)$; a decrease in the organ indexes of the kidney, spleen and adrenal gland $(P<0.01)$; an increase in the organ index of the thymus $(P<0.05)$; a decrease in the levels of T3, T4, FT4, cAMP and in the ratio of cAMP/cGMP $(P<0.01, P<0.05)$. And in the same experiments, compared with those in the RART group, the rats in the SSART group showed a decrease in the level of FT3 $(P<0.01)$, an increase in the level of TSH $(P<0.01)$; and no obvious change in the level of CGMP $(P>0.05)$. In addition, in the same experiments, compared with those in the $B C$ group, the rats in the RYDS model group showed a decrease in the mRNA and protein expressions of PI3K, AKT and $m$ TOR $(P<0.01)$; compared with those in the RYDS model group, the rats in both the RART group and the SSART group showed an increase in the mRNA expression of PI3K, AKT and mTOR in the kidney $(P<0.01)$; compared with those in the RART group, the rats in the SSART group showed an increase in the level of the protein expression of PI3K, AKT and mTOR in the kidney $(P<0.01, P<0.05)$, among which, the level of the protein expression of PI3K and mTOR was higher than that done by the rats in the RART group $(P \otimes 0.05)$. The above-mentioned results imply that in the relevant experiments, the RYDS-treating effect of the salt stir-fried AR was significantly stronger than that of the raw AR in the rats with RYDS.

The data of the RYDS-treating effect of the suspension of mangiferin powder in the rats with RYDS are arranged in Fig. 10-13. It can be seen that in the relevant experiments, after 14 days of intragastric administration of the suspension of mangiferin powder, compared with those in the RYDS model group, the rats in the MPT group showed an increase in the variation of body weight and pain threshold $(P<$ 0.01); a decrease in anal temperature, water-intake, food-intake, urine volume, and the organ indexes of the kidney, spleen, and adrenal gland $(P<0.01)$; an increase in the organ index of the thymus $(P<0.05)$; a decrease in the levels of T3, T4, FT3, FT4, cAMP, and in the ratio of cAMP/cGMP $(P<0.01)$; an increase in the level of TSH $(P<0.01)$; and no obvious change in the level of cGMP $(P>0.05)$. In addition, in the same experiments, compared with those in the BC group, the rats in the RYDS model group showed a decrease in the levels of the mRNA and protein expressions of PI3K, AKT and mTOR $(P<0.01)$; and compared with those in the RYDS model group, the rats in the MPT group showed an increase in the levels of the mRNA and protein expressions of PI3K, AKT and mTOR in the kidney $(P<0.01, P<0.05)$. The above-mentioned results imply that in the relevant experiments, the suspension of mangiferin powder exerted a very good RYDS-treating effect in the rats with RYDS.

\section{Discussion}

AR is a TCM herb frequently used to treat RYDS. It has been proved by the TCM clinical experiences that the RYDS-treating effect of raw AR can be strengthened by salt stir-frying. In TCM, RYDS is considered to manifest a series of symptoms, such as low fever, sore waist and knees, dizziness and tinnitus, feverish dysphoria, emaciation and weakness, tidal fever and night sweats, and spermatorrhea[31, 32]. In modern 
medicine, RYDS is one of the commonest TCM syndromes considered to be with the so-called sub-health state[33, 34]. Moreover, certain common diseases in modern medicine, such as hypertension[35, 36], diabetes[37, 38], coronary heart disease[39, 40], and menopausal syndrome[41], can also be found to have a relation with RYDS. Therefore, it is of great significance to carry out pharmaceutical and pharmacological researches on the RYDS-treating effect of AR.

In our experiments on the RYDS-treating effect of AR, the thyroxine-induced rat RYDS model[42]was adopted. During RYDS modeling with the rats, compared with those in the BC group, the rats in the RYDS model group manifested abnormal outward appearance and behavior, and abnormalities of various detected indexes (see Fig. 7-9). The RYDS-related abnormalities detected in the RYDS model rats mainly included a decrease in the variation of body weight and pain threshold $(P<0.01)$; an increase in anal temperature, water-intake, food-intake and urine volume $(P<0.01)$; an increase in the organ indexes of the kidney, adrenal gland and spleen $(P<0.01)$; a decrease in the organ index of the thymus $(P<0.01)$; an increase in the levels of T3, T4, FT3 and FT4 $(P<0.01)$; an increase in both the level of cAMP and the ratio of CAMP/CGMP $(P<0.01)$; a decrease in the level of TSH $(P<0.01)$; and a decrease in the level of cGMP $(P<0.05)$. In our experiments, Liuwei Dihuang Pills and propylthiouracil tablets, 2 frequently-used effective RYDS-treating preparations, were chosen as the positive control agents. Compared with those in the BC group, the rats in the LDPT group and PTT group showed a satisfactory RYDS-treating result of the 2 positive control agents (see Fig. 7-8). In our experiments, when raw AR and salt stir-fried AR were used to treat the rats with RYDS, we found that, compared with the 2 positive control agents, both of raw AR and salt stir-fried AR showed a satisfactory RYDS-treating effect. The data of the detections of various indexes (see Fig. 10-13) showed that both raw AR and salt stir-fried AR exerted their RYDStreating effect by adjusting or restoring RYDS-affected general physiological indexes or endocrinoimmunological indexes, such as increasing the variation of body weight and pain threshold $(P<0.01)$; decreasing anal temperature, water-intake, food-intake and urine volume $(P<0.01)$; reducing the organ indexes of the kidney, spleen and adrenal gland $(P<0.01)$; elevating the organ index of the thymus $(P<$ 0.05); lowering the levels of T3, T4, FT3, FT4,CAMP and the ratio of cAMP/cGMP $(P<0.01 ; P<0.05)$; increasing the levels of TSH and cGMP $(P<0.01 ; P<0.05)$; and elevating the mRNA and protein expressions of PI3K/AKT/mTOR pathway (P凶0.01), which imply that the mechanism of the RYDS-treating effect of both raw AR and salt stir-fried AR is just the same as that of the 2 positive control agents.

We found in our experiments that both raw AR and salt stir-fried AR could exert RYDS-treating effect, and that the effect of the salt stir-fried AR was stronger than that of raw AR (see Fig. 10-13); and we also found that neomangiferin and mangiferin were the principal chemical components in both raw AR and salt stir-fried $A R$, and that compared with those in the raw AR, the content of neomangiferin became decreased and that of mangiferin became increased in the salt stir-fried AR (see Fig. 2-4,Tab. 2), which was in accordance with the results from the previous researches done by other scholars[20, 21, 22, 23]. According to these experimental results we inferred that mangiferin was possibly the material basis of the RYDS-treating effect of both raw AR and salt stir-fried AR. The results from our experiment on the RYDS-treating effect of mangiferin (see Fig. 10-13) showed that, just as what the raw AR and salt stirfried AR did, mangiferin could also increase the variation of body weight and pain threshold $(P<0.01)$; 
decrease anal temperature, water-intake, food-intake, urine volume, and the organ indexes of the kidney, spleen, and adrenal gland $(P<0.01)$; increase the organ index of the thymus $(P<0.05)$; lower the levels of T3, T4, FT3, FT4, CAMP, and the ratio of cAMP/cGMP $(P<0.01)$; elevate the levels of TSH $(P<0.01)$ and the mRNA and protein expressions of PI3K/AKT/mTOR pathway in the kidney of the rats with RYDS $(\mathrm{P}<$ $0.01, \mathrm{P}<0.05)$, which implies that our inference is reasonable. And at the same time, the results from our experiments also imply that the cause of the enhancement of the RYDS-treating effect of the salt stir-fried $A R$ is just the increase in the content of mangiferin, and that there is a possible dose-effect relationship between mangiferin and the RYDS-treating effect.

The results of the HPLC determination (see Fig. 2-3) show that the peaks of mangiferin in the chromatograms of salt stir-fried AR and water stir-fried AR are higher and the peaks of neomangiferin are lower, compared with those in the chromatogram of raw AR, which implies there was a change in the contents of neomangiferin and mangiferin in raw AR after being stir-fried in water or in salt, which could be expressed by 2 sequences: for the content of mangiferin, salt stir-fried $A R>$ water stir-fried $A R>$ raw $A R$, and for the content of neomangiferin, salt stir-fried $A R<$ water stir-fried $A R<$ raw AR. The amounts of neomangiferin and mangiferin calculated by using the relative peak area method (see Table 2) can verify these 2 sequences, which is in accordance with the results from the previous researches done by other scholars[20, 21, 22, 23]. Therefore, we inferred that the change in the contents of neomangiferin and mangiferin in raw AR after being stir-fried in salt or in water was just because there was a possible neomangiferin-mangiferin transformation induced by the factors of salt, temperature and water during the salt (or water) stir-frying of raw AR. In order to certify the rationality of our inference, we prepared the simulative salt stir-fried neomangiferin and simulative neomangiferin decoction slices (see Fig. 1). The HPLC chromatograms of the simulative salt stir-fried neomangiferin (see Fig. 5) show that before being simulatively stir-fried in salt, neomangiferin exhibits only 1 peak, which represents neomangiferin, and however, after being simulatively stir-fried in salt, neomangiferin exhibits 2 peaks, the newly-appeared one of which, represents mangiferin. Similarly, the HPLC chromatograms of the salt stir-fried simulative neomangiferin decoction slices (see Fig. 6) also show 2 peaks, the lower one of which represents neomangiferin, and the higher one of which represents mangiferin. The results the 2 HPLC determinations imply that the salt stir-frying can induce a neomangiferin-mangiferin transformation, which caused the content of neomangiferin to decrease and the content of mangiferin to increase in AR.

We may speculate that key point of the neomangiferin-mangiferin transformation was the hydrolysis of the 7-0-glc bond in the chemical construction of neomangiferin under the influence of certain factors from the salt (or water) stir-frying process, such as heating, sodium chloride, $\mathrm{cl}^{-1}$-radical, water, etc. (see Fig. 14.)

In our present research, concerning the RYDS-treating effect of AR, only the neomangiferin and mangiferin in AR were studied; and the influence of other chemical components in AR on the RYDStreating effect of $A R$, the actual process of the neomangiferin-mangiferin transformation during the salt stir-frying of raw AR, and the possible dose-effect relationship between mangiferin and the RYDS-treating effect will be studied in future.

Page $17 / 34$ 


\section{Conclusion}

The material basis and mechanism of the RYDS-treating effect of raw AR and salt stir-fried AR, the cause of the change in the contents of neomangiferin and mangiferin in raw AR after being stir-fried in salt or in water, and the cause of the enhancement of the RYDS-treating effect of salt stir-fried AR, were investigated in the present paper. Based on the results of our investigation, we may conclude that (1) mangiferin may be the material basis of the RYDS-treating effect of both raw AR and salt stir-fried AR; (2) raw AR and salt stir-fried AR exert their RYDS-treating effect by adjusting or restoring some RYDSaffected general physiological and endocrino-immunological indexes, such as increasing the variation of body weight and pain threshold; decreasing anal temperature, water-intake, food-intake and urine volume; reducing the organ indexes of the kidney, spleen and adrenal gland; elevating the organ index of the thymus; lowering the levels of T3, T4, FT3, FT4; cAMP and the ratio of cAMP/cGMP; increasing the levels of TSH and cGMP; and elevating the mRNA and protein expressions of PI3K/AKT/mTOR pathway; (3) the salt (or water) stir-frying may induce a neomangiferin-mangiferin transformation in $A R$, which causes the content of neomangiferin to decrease and the content of mangiferin to increase in AR; (4) the cause of the enhancement of the RYDS-treating effect of salt stir-fried AR may be the increase in the content of mangiferin; and (5) the technological theory of the salt stir-frying of AR may be considered as the enhancement of its RYDS-treating effect by increasing the content of mangiferin.

\section{Abbreviations}

AR

Anemarrhenae Rhizoma; BC:Blank Control ; cAMP:Cyclic Adenosine Monophosphate; cGMP:Cyclic Guanosine Monophosphate; FT3:Free Triiodothyronine; FT4:Free Tetraiodothyronine; HPLC:High Performance Liquid Chromatography; HRP:Horseradish Peroxidase; LDPT:Liuwei Dihuang Pills Treating; MPT:Mangiferin Powder Treating; PCA:Principal Components Analysis; PCR:Real-Time Polymerase Chain Reaction; T3:Triiodothyronine; PTT:Propylthiouracil Tablets Treating; RART:Raw AR Treating; RYDS:renal yin deficiency syndrome; SSART:Salt Stir-fried AR Treating; T4:Tetraiodothyronine; TCM:Traditional Chinese Medicine; TSH:Thyroid Stimulating Hormone.

\section{Declarations}

\section{Acknowledgement}

The authors wish to thank Prof. Yilun Song of Beijing University of Chinese Medicine for his help in revision and modification of the manuscript of this paper.

\section{Authors' Contributions}

SH and JKX participated in the design of research program and the manuscript drafting of this paper, and were responsible for the performance of the experiments and the data analysis concerning the simulative 
salt stir-frying of neomangiferin, and the salt stir-frying and the RYDS-treating effect of AR; WFL, XTH and ZQL participated in the performance of the experiments on the salt stir-frying and the RYDS-treating effect of AR; MRZ participated in the performance of the experiment on the RYDS-treating effect of mangiferin; KYL, YNT and HYW participated in the performance of the experiments on the simulative salt stir-frying of neomangiferin, and the salt stir-frying and the RYDS-treating effect of AR; XYL collected the needed literature and information related to this research program; ZGY participated in part of the data analysis concerning the relevant experiments; $H D$ and $F L$ were responsible for the design of the research program and the supervision of the performance of the relevant experiments. SH and HD prepared the final version of the manuscript. All authors read and approved the final manuscript.

\section{Funding}

This publication was supported by the National Natural Science Foundation of China (NSFC, 81774004); National Key Research and Development Plan (2016YFE0129000); Beijing Municipal Natural Science Foundation (BJNSF, 7182093); the National Key Research and Development Plan (2018YFC1706303).

\section{Availability of data and materials}

The datasets used in this study are available from the corresponding author upon reasonable request.

\section{Ethics approval and consent to participate}

Experiments were approved by the Experimental Animal Ethics Committee of Beijing University of Chinese Medicine, Beijing. Consent to participate is not relevant to this manuscript.

\section{Consent for publication}

The manuscript is approved by all authors for publication.

\section{Competing Interests}

All the authors declare that they have no competing interests.

\section{References}

1. Chinese Pharmacopeia Commission. Pharmacopeia of the People's Republic of China (2015-edition) Part I. Beijing: China Medical Science and Technology Publishing House; 2015. pp. 212-3. 
2. Wu P. (Wei Dynasty). Shennong's Classic of Materia Medica. Shanghai: The Commercial Press; 1955. p. 67.

3. Zhao CC, Wei F, Zhang JQ. Progress in research of pharmacological actions of Anemarrhena asphodeloides. Chin J New Drugs Clin Rem. 2015;34(12):898-902. doi:10.14109/j.cnki.xyylc.2015.12.002.

4. 10.3969/j.issn.1674-7860.2017.12.063

Feng F. A review on pharmcological effects of Rhizoma Anemarrhenae. CJCM. 2017;9(12): 133, 137. doi:10.3969/j.issn.1674-7860.2017.12.063.

5. Hu HY, Zhang R, Zhang YF, et al. Role of CREB in the regulatory action of sarsasapogenin on muscarinic M1 receptor density during cell aging. FEBS Lett. 2010;584(8). doi:10.1016/j.febslet.2010.03.006.

6. Lee B, Jung K, Kim DH, et al. Timosaponin A-III, a saponin isolated from Anemarrhena asphodeloides, ameliorates learning and memory deficits in mice. 2009;93(2):121. doi:10.1016/j.pbb.2009.04.021.

7. Oh JK, Hyun SY, Oh HR, et al. Effects of Anemarrhena asphodeloides on focal ischemic brain injury induced by middle cerebralartery occlusion in rats. Biol Pharm Bull. 2007;30(1):38. doi:10.1248/bpb.30.38.

8. Wang GJ, Lin LC, Chen CF, et al. Effect of timosaponin A-III, from Anemarrhenae asphodeloides Bunge (Liliaceae), on calcium mobilization in vascular endothelial and smooth muscle cells and on vascular tension. Life Sci. 2002;71(9):1081.

9. Xiao S, Xu M, Ge Y, et al. Inhibitory effects of saponins from Anemarrhena asphodeloides bunge on the growth of vascular smooth muscle cells. Biomed Environ Sci. 2006;19(3):185.

10. Peng ZG, Yao YB, Yang J, et al. Mangiferin induces cell cycle arrest at G2/M phase through ATR-Chk1 pathway in HL-60 leukemia cells. Genet Mol Res. 2015;14(2):4989-5002.

doi:10.4238/2015.May.12.2.

11. Wei ZQ, Deng JG, Yan L, et al. The anti-inflammatory actions of mangiferin on the chronic inflammation induced by lipopolysaccharide. Pharmacol Clin Chin Mater Med. 2011;27(02):43-5. doi:10.13412/j.cnki.zyyl.2011.02.017.

12. Zhang J, Han YP, Luo M. Different mRNAs in Type 2 Diabetes Mellitus within Kidney-Yin Deficiency. Lishizhen Med Mater Med Res. 2017;28(11):2798-800. doi:10.3969/j.issn.1008-0805.2017.11.087.

13. Dou C. Bianque Xinshu. Beijing: China Medical Science and Technology Publishing House; 2011. pp. 19-21.

14. Wu Q. (Qing Dynasty). Yizong Jinjian. 337. Beijing: People's Medical Publishing House; 2006.

15. Yan L, Wang XT, Gao H. Effect of Ethanol Extract from Saline Rhizoma Anemarrhenae on Blood Glucose and Blood Lipid in Rat Mellitus. China Pharm. 2019;28(10):1-3. doi:10.3969/j.issn.10064931.2019.10.001.

16. Zhang S, Wang XT, Gao Y, et al. Effects of the active fractions in salt processed Rhizoma Anemarrhenae on insulin resistance. Chin J Hosp Pharm. 2017;37(21):2127-30. doi:10.13286/j.cnki.chinhosppharmacyj.2017.21.05. 
17. Sun YN, Liu Z, Feng W. Essential empirical study of the deficieny of kindey-yin in diabetes mellitus based on metabonomics. J Chengdu Univ Tradit Chin Med. 2009;32(02):69-71. doi:10.13593/j.cnki.51-1501/r.2009.02.004.

18. Wang LK, Cui WF, Pan YY, et al. Comparison of Distribution Characteristics of TCM Syndrome Types in Hypertension Patients with Different Body Mass Index. Liaoning J Tradit Chin Med. 2018;45(06):1211-3. doi:10.13192/j.issn.1000-1719.2018.06.030.

19. Wang T, Zhang W, Luo Y, et al. Curative Effect of Compound Qishao Jiangya Tablets in Patients with Yin Deficiency of Liver and Kidney Type Hypertension and Its Influences on Levels of Ang-ânLand ANP. Liaoning J Tradit Chin Med. 2018;45(06):1225-7. doi:10.13192/j.issn.1000-1719.2018.06.034.

20. Liu M, Zhao BY, Zhao L, et al. Changes of mangiferin and neomangiferin contents in Rhizoma Anemarrhenae before and after processing. Acad J Second Mil Med Univ. 2006;(05): 528-30. doi:10.16781/j.0258-879x.2006.05.017.

21. Su XN, Ji D, Zhou YP, et al. Simultaneous determination of flavones and saponins of Rhizoma Anemarrhenae by HPLC-DAD-ELSD. China J Chin Mater Med. 2015;40(01):108-11. doi:10.4268/cjcmm20150121.

22. Song ZB, Wu Y, Gao H. Determination of neomangiferin, mangiferin, and isomangiferin in Anemarrhenae Rhizoma before and stir-frying with salt-water by HPLC. Drugs Clin. 2015;30(02):1458. doi:10.7501/j.issn.1674-5515.2015.02.007.

23. Ji D, Huang ZY, Fei CH. Comprehensive profiling and characterization of chemical constituents of rhizome of Anemarrhena asphodeloides Bge. J CHROMATOGR B. 2017;1060. doi:10.1016/j.jchromb.2017.06.032.

24. Wu Y, Song ZB, Xu Y, et al. Comparison of Nourishing Yin Effect of Anemarrhenae Rhizoma before and after Processing. Chin J Exp Tradit Med Formulae. 2013;19(24):211-4. doi:10.11653/syfj2013240211.

25. Liu YJ, Du JY, Huang SP. Experience of HUANG Suping in the Treating Perimenopausal Hot Flashes. Clin J Tradit Chin Med. 2017;29(08):1227-9. doi:10.16448/j.cjtcm.2017.0408.

26. Li WF, Xu JK, Zhi MR, et al. Optimization of Rhizoma Anemarrhenae stir-frying with salt-water process based on orthogonal design, principal component analysis and analytic hierarchy. China $J$ Tradit Chin Med Pharm. 2018;33(09):4167-70.

27. Ma YM, Liu MY, Chen Q, et al. Advances in Pharmacological Effects of Mangiferin on Metabolic Diseases. Journal of Changchun University of Chinese Medicine. 2019;35(03):606-9. doi:10.13463/j.cnki.cczyy.2019.03.057.

28. Wei ZQ, Yan L, Deng JG. Effects of mangiferin on MAPK pathway and serum cytokines in rats with chronic inflammation induced by lipopolysaccharide. Chin. Tradit. Herb. Drugs. 2013;44(01):52-58. doi:0253-2670 (2013) 01-0052-07.

29. Qin LP, Shi HP, Zheng SQ, et al. Effects of osthol and icariin on serum thyroid hormone in mice of hypothyroidism type kidney-yang insufficiency. Acad J Sec Mil Med Univ. 1998;(01):49-51. doi:10.16781/j.0258-879x.1998.01.024. 
30. Tan JH, Li HM, Li H, et al. Effect of Different Dosage of Liuwei Dihuang Pill on Lipid Metabolism, Adiponectin and Insulin Resistance of Gestational Diabetic Rats. China J Chin Med. 2019;34(08):1686-90. doi:10.16368/j.issn.1674-8999.2019.08.398.

31. Bai R, Liu XX, Li FJ, et al. Modeling method of animal models with Yin deficiency syndrome. Chin Arch Tradit Chin Med. 2020;38(02):69-71. doi:10.13193/j.issn.1673-7717.2020.02.019.

32. Lin Y, Zhang T, Li X, et al. Research overview of animal models with a kidney-deficient zheng of traditional Chinese medicine. Chin Arch Tradit Chin Med. 2020;38(01):136-9. doi:10.13193/j.issn.1673-7717.2020.01.032.

33. Zhang JD, Sun GX, He QH. Characteristics of TCM syndromes and influential factors of Sub-health status among Hunan university students. J Tradit Chin Med Univ Hunan. 2014;34(03):54-7. doi:10.3969/j.issn.1674-070X.2014.03.016.054.04.

34. Wei M, Zhao XS, Sun XM, et al. Comparative study on gene expression of kidney-Yin deficiency and kidney-Yang deficiency. J. Shanxi Med. Univ. 2012;43 (09): 652-654, 722. doi:1007-6611 (2012) 090652-03.

35. Dou JY, Zhou HJ, Du YL. Clinical Efficacy Observation of Combination of Qiju Dihuang Pill and Sustained-release Nifedipine on Hypertension of Liver-Kidney Yin Deficiency Type. J Liaoning Univ Tradit Chin Med. 2019;21(06):147-9. doi:10.13194/j.issn.1673-842x.2019.06.039.

36. Qu XY, Yang XD, Yao FS. Treatment and TCM Pathogenesis of "Deficiency in Nature and Excess in Superficiality"for Primary Hypertension. Acta Chin Med Pharmacol. 2018;46(06):10-2. doi:10.19664/j.cnki.1002-2392.180169.

37. Jin HJ, Qiu KC, Li JH, et al. The improving effect of Zhimu-Huangbai herb pair on insulin resistance. CPB. 2019;35(07):1020-4. doi:10.3969/j.issn.1001-1978.2019.07.025.

38. Zhang C, Wang JS, Zhang GB, et al. Protective effects of mangiferin on the diabetic nephropathy in streptozotocin-induced diabetic rats. Acta Acad Med Wannan. 2017;36(04):314-7. doi:10.3969/j.issn.1002-0217.2017.04.003.

39. Liu XX, Li T, Li HY, et al. Shenqi Gualou Xiebai Banxia Decoction for the treatment of 50 cases of hepatic and kidney yin deficiency coronary atherosclerotic heart disease with diabetes. Global Tradit Chin Med. 2019;12(12):1871-4. doi:10.3969/j.issn.1674-1749.2019.12.022.

40. Lv Y, Wang ZL. Correlation between TCM syndrome types and the degree of coronary vascular lesions in patients with coronary artery disease. J Liaoning Univ Tradit Chin Med. 2017;19(10):199202. doi:10.13194/j.issn.1673-842x.2017.10.061.

41. Quan XM, Xu LW, Song CX, et al. The clinical efficacy of Ziyin Pinggan Bushen decoction and acupuncture treatment in liver and kidney Yin deficient perimenopausal syndrome. J Southeast Univ Med Sci Ed. 2019;38(04):672-6. doi:10.3969/j.issn.1671-6264.2019.04.021.

42. Sun JF. Methodology of Animal Experiments. Beijing: People's Medical Publishing House; 2001. p. 488.

\section{Tables}


Table 1 Primer Sequences for Real-Time RT-PCR

\begin{tabular}{|ll|}
\hline Primer Names & Primer Sequences \\
\hline PI3K & Forward:5'-TGCTATGGCAGACACCCTTG-3' \\
\cline { 2 - 2 } & Reverse:5'-CTTCCCGGGGTACTTCCAAC-3' \\
AKT & Forward:5'-ACAGGTCGCTACTATGCCATGAAGA-3' \\
\hline mTOR & Reverse:5'-GCAGGACACGGTTCTCAGTAAGC-3' \\
& Forward:5'-TGTGGCAAGAGCGGCAGACT-3' \\
\hline GAPDH & Foverse:5'-TGTGGCAAGAGCGGCAGACT-3' \\
& Reverse:5'-CGCCAGTAGACTCCACGACA-3' \\
\hline
\end{tabular}

Table 2 Results of the HPLC Determination of the contents of Neomangiferin and Mangiferin in Raw AR, Salt stir-fried AR and Water Stir-fried AR $(x \pm s, n=3)$

\begin{tabular}{|llll|}
\hline Samples & $\begin{array}{l}\text { Mangiferin } \\
(\%)\end{array}$ & $\begin{array}{l}\text { Neomangiferin } \\
(\%)\end{array}$ & $\begin{array}{l}\text { Value of Ratio of } \\
\text { Mangiferin / Neomangiferin }\end{array}$ \\
\hline Raw AR & $0.64 \pm 0.05$ & $2.41 \pm 0.05$ & 0.27 \\
\hline Water Stir-fried AR & $0.85 \pm 0.06$ & $1.68 \pm 0.05$ & 0.51 \\
\hline Salt Stir-fried AR & $0.97 \pm 0.06$ & $1.21 \pm 0.03$ & 0.80 \\
\hline
\end{tabular}

Note: The value of the ratio of mangiferin / neomangiferin represents the magnitude of the amount of mangiferin in a sample.

\section{Figures}


A

Preparation of "Blank AR Decoction Slices"

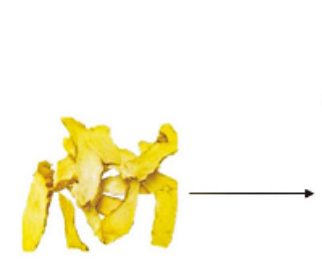

AR decoction slices

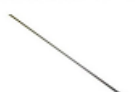

percolation device

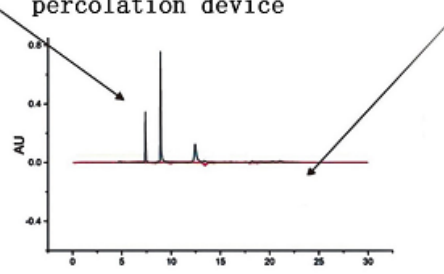

B

Salt Stir-frying of the Simulative Neomangiferin Decoction Slices

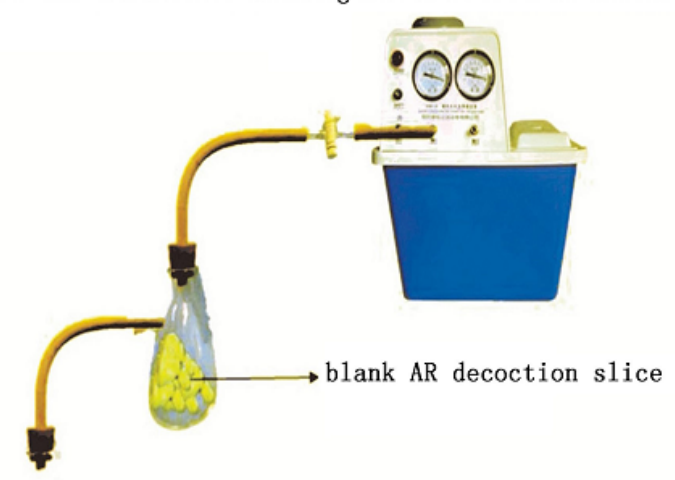

$\longrightarrow$ Neomangiferin

\section{Figure 1}

Schematic Diagram of the Salt Stir-frying of Simulative Neomangiferin Decoction Slices Notes: $A=$ preparation of "blank AR decoction slices", and B = salt stir-frying of the simulative neomangiferin decoction slices.

A

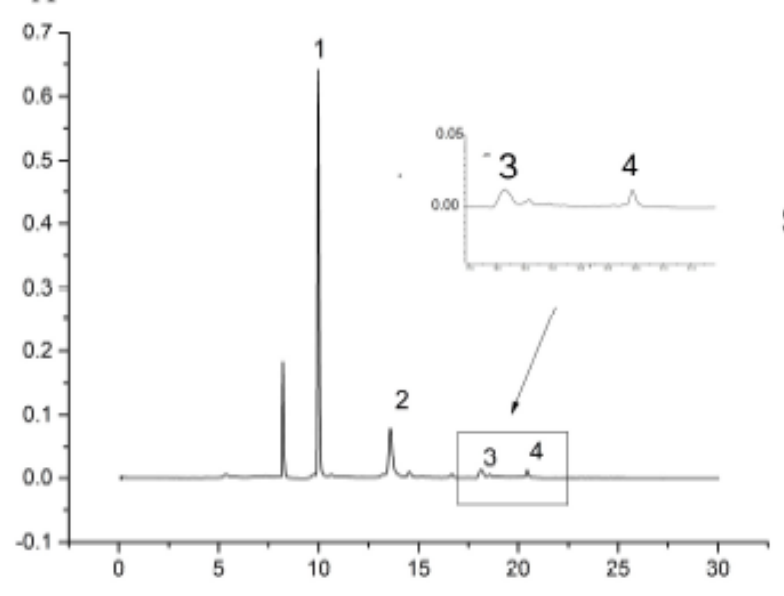

B

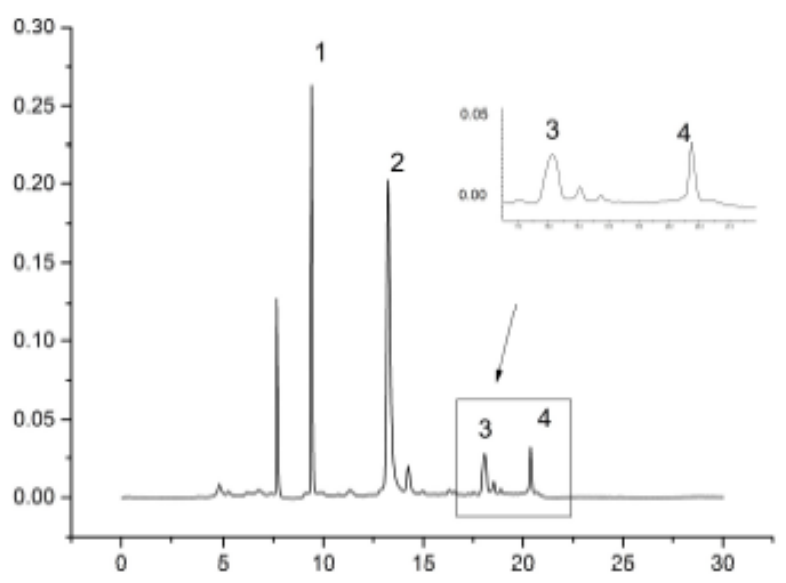

Figure 2

HPLC Chromatograms of the Principal Chemical Components of Raw AR and Salt Stir-fried AR Notes: A = raw $A R$. $B=$ salt stir-fried AR. 1 = neomangiferin, $2=$ mangiferin, $3=$ Icariin, and $4=$ anemarrhenasaponin BII. 


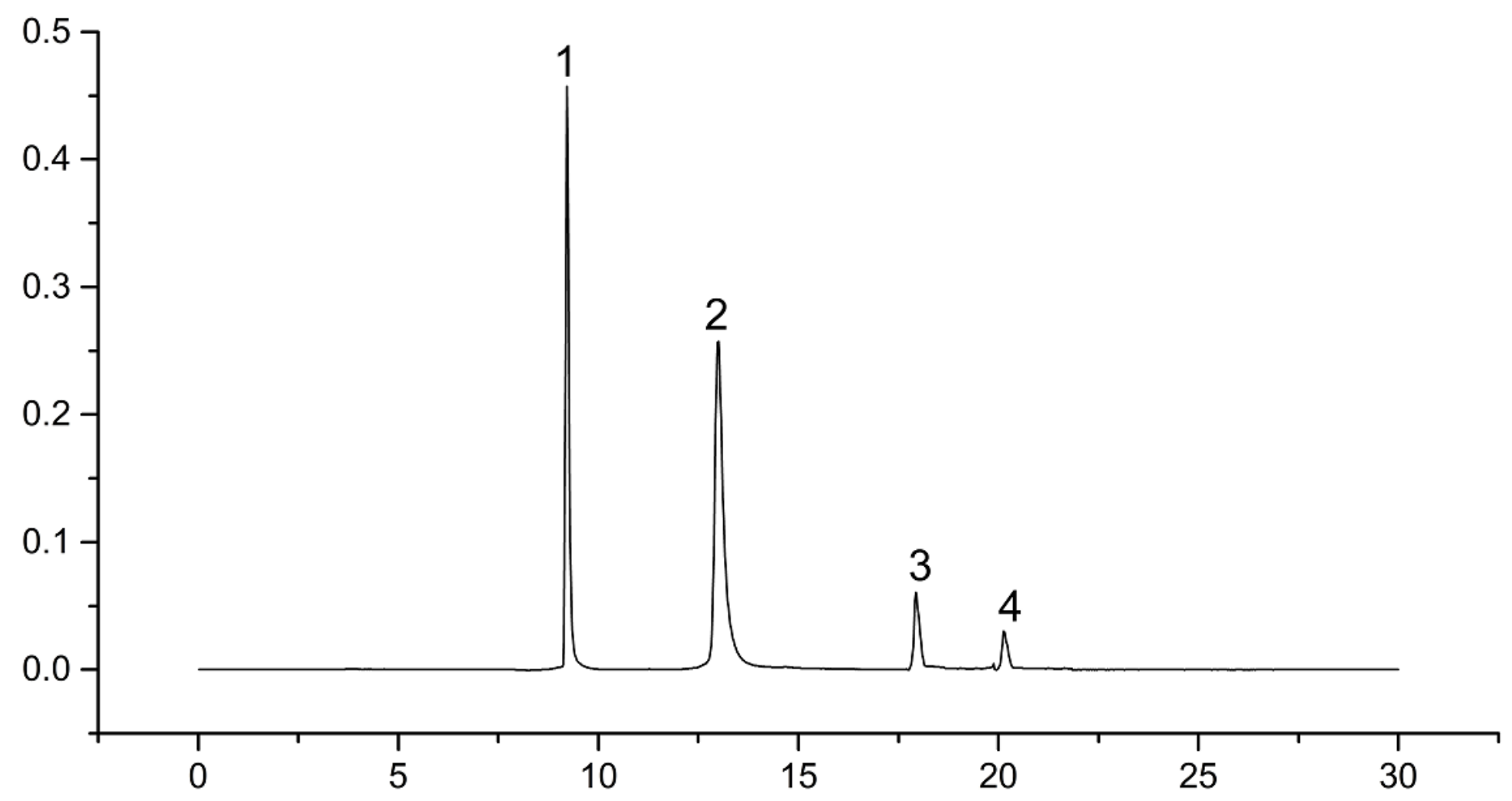

Figure 3

HPLC Chromatograms of the Principal Chemical Components of Water Stir-fried AR Notes: $1=$ neomangiferin, 2 = mangiferin, 3 = Icariin, and 4 = anemarrhenasaponin Bll.

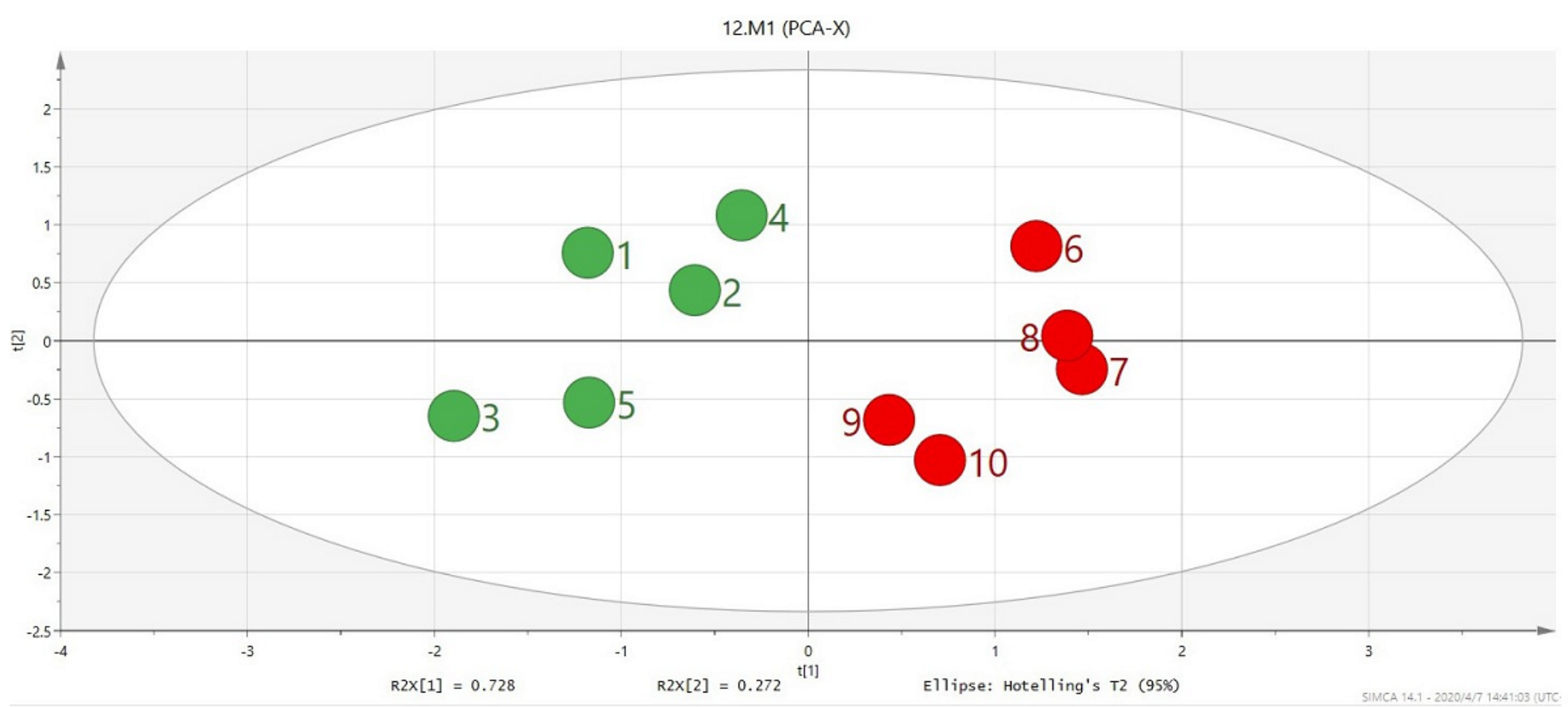

Figure 4 
PCA Score Plot of Salt Stir-fried AR and Raw AR Notes: the green markers (1-5) = salt stir-fried AR, and the red markers (6-10) $=$ raw AR.
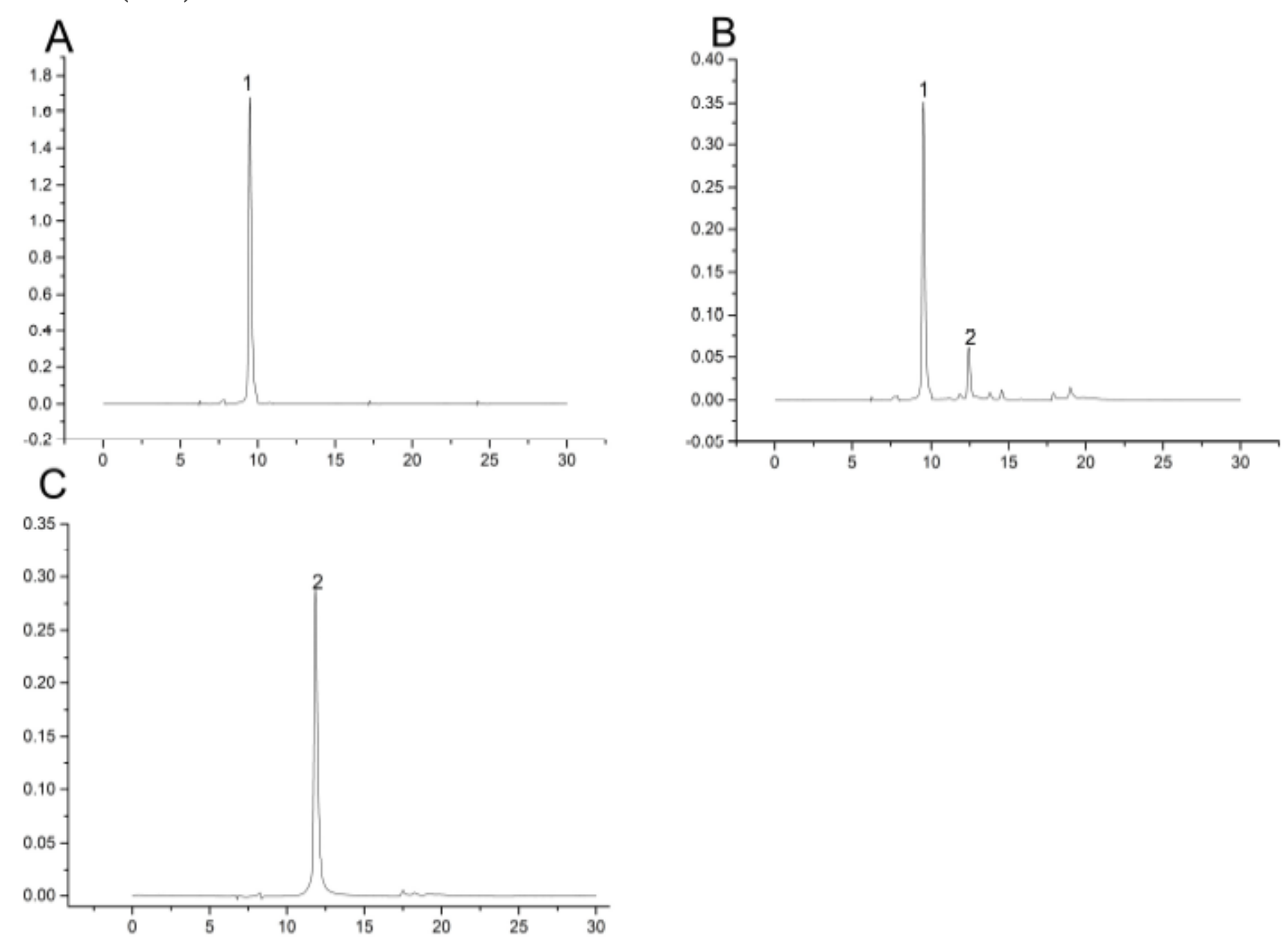

\section{Figure 5}

HPLC Chromatograms of Simulative Salt Stir-fried Neomangiferin Notes: A = HPLC chromatogram of neomangiferin before being simulatively stir-fried in salt, $B=$ HPLC chromatogram of neomangiferin after being simulatively stir-fried in salt, $C=$ HPLC chromatogram of mangiferin, $1=$ neomangiferin, and $2=$ mangiferin. 

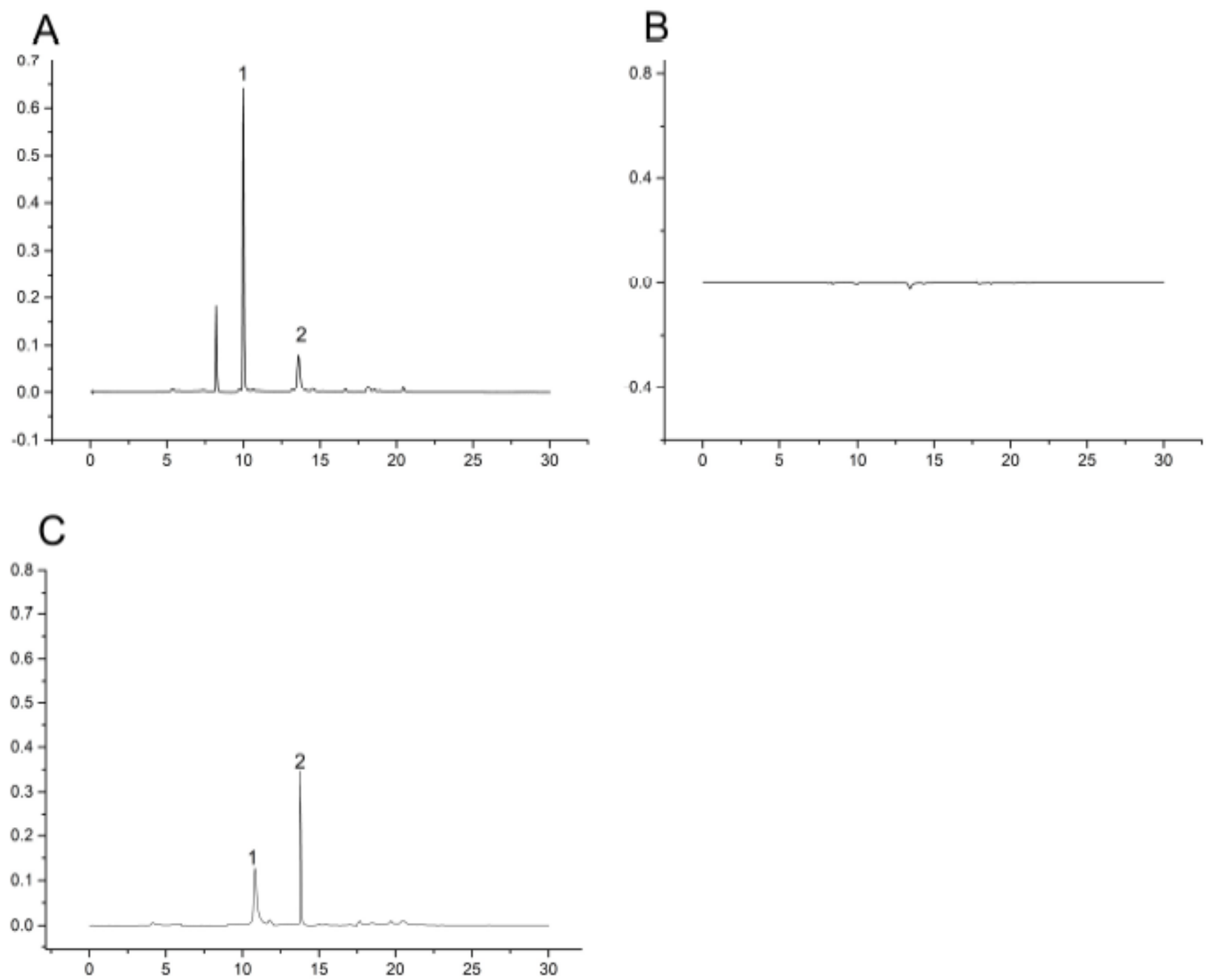

Figure 6

HPLC Chromatogram of the Salt Stir-fried Simulative Neomangiferin Decoction Slices Notes: $A=$ HPLC chromatogram of raw AR, B = HPLC chromatogram of "blank AR decoction slices", C = HPLC chromatogram of salt stir-fried simulative neomangiferin decoction slices, $1=$ neomangiferin, and $2=$ mangiferin. 

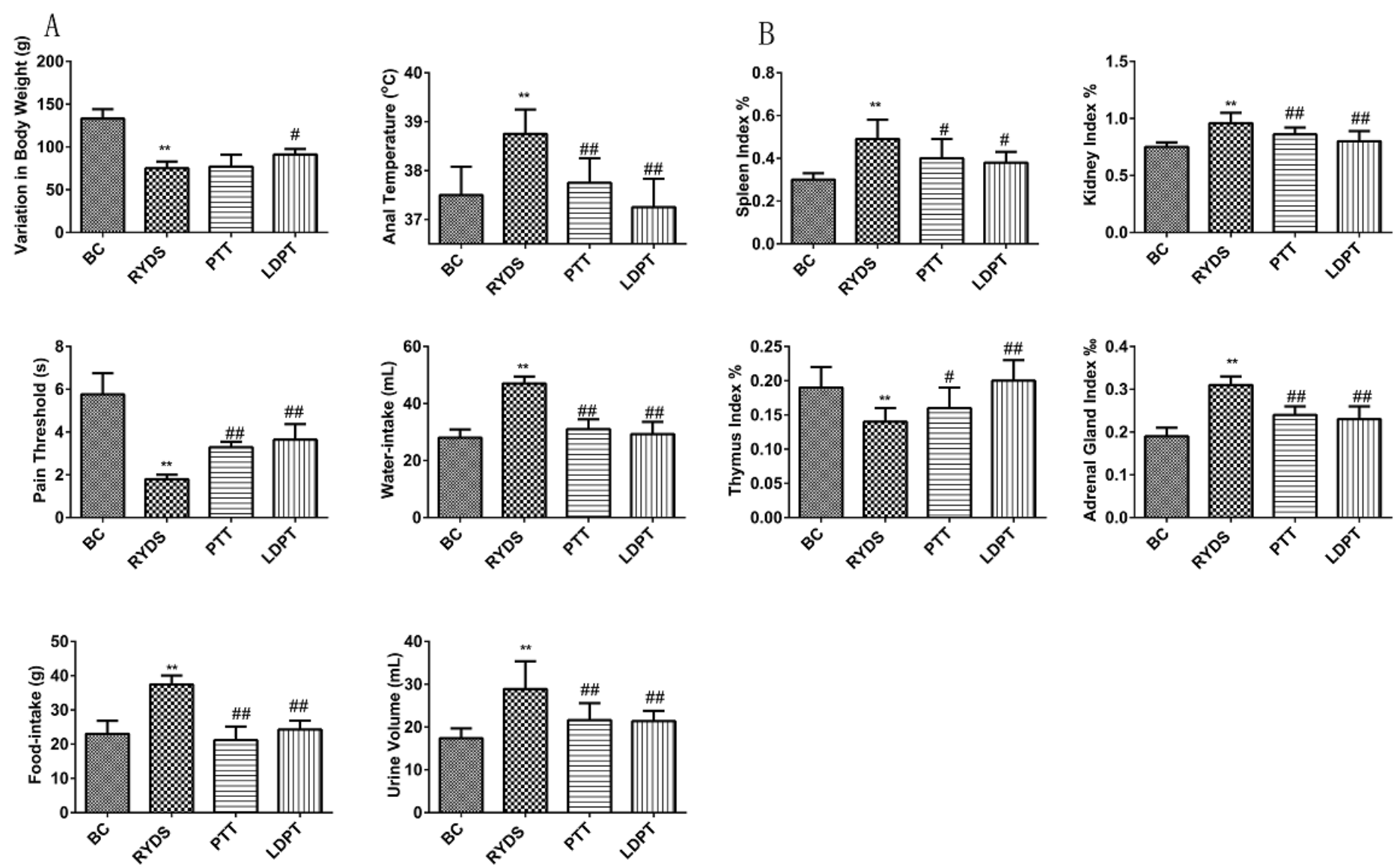

Figure 7

Effects of Propylthiouracil Tablets and Liuwei Dihuang Pills on Physiological Indexes and the Organ Indexes of Endocrine-immunological Organs of the Rats with RYDS. A = Data of the detection of physiological indexes. $B=$ Data of the detection of the organs indexes of endocrine-immunological organs. All the data are expressed as the means $\pm S D(n=8) .{ }^{*}$ Compared with $B C$ group, $P<0.05$; **compared with BC group, $\mathrm{P}<0.01$; \# Compared with RYDS model group, $\mathrm{P}<0.05$; and \#\# compared with RYDS model group, $\mathrm{P}<0.01$. 

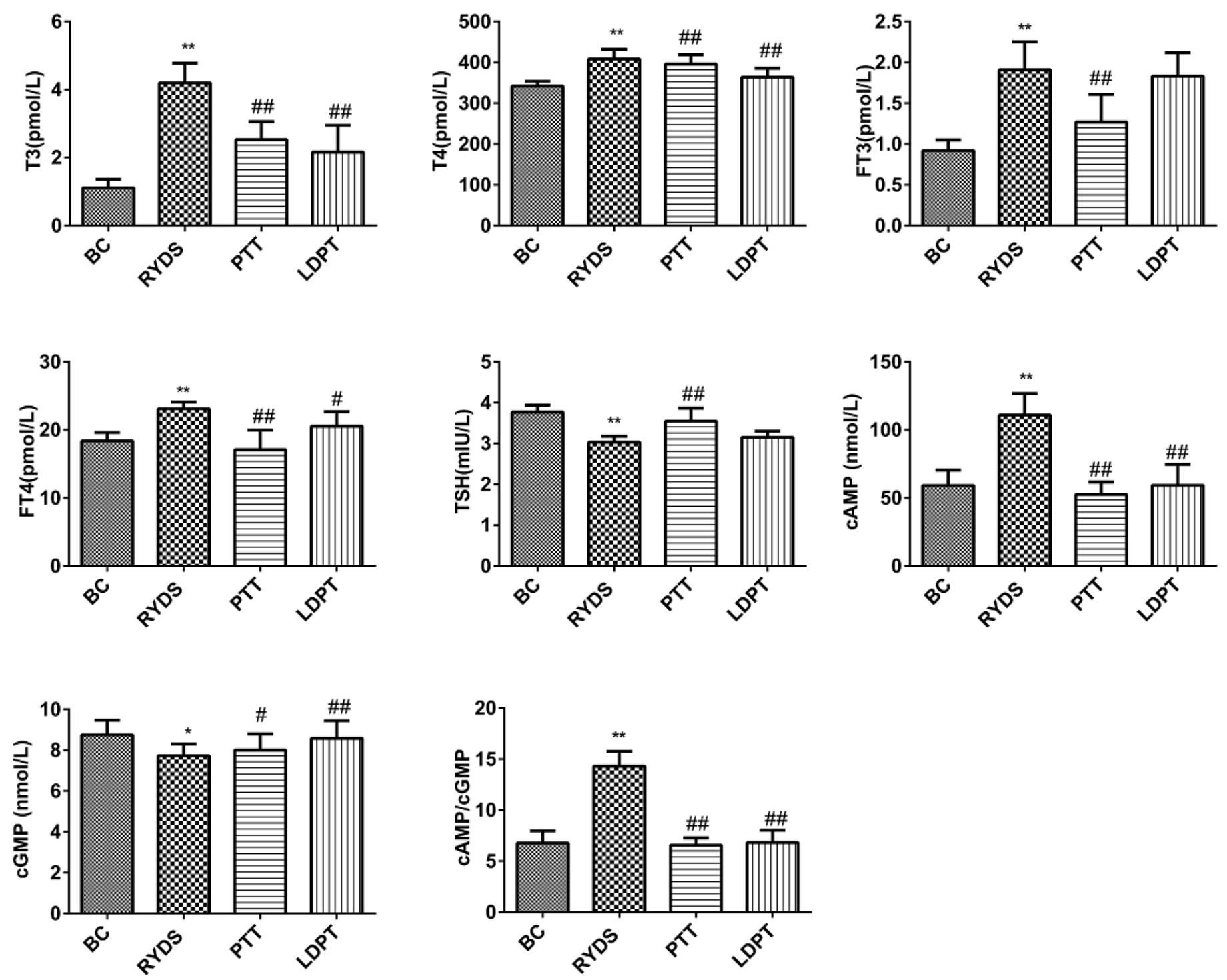

Figure 8

Effects of Propylthiouracil Tablets and Liuwei Dihuang Pills on Endocrine-immunological Indexes in the Serum or Plasma of Rats with RYDS. All the data are expressed as the means $\pm S D(n=8)$. ${ }^{*}$ Compared with $\mathrm{BC}$ group, $\mathrm{P}<0.05$; ${ }^{*}$ compared with $\mathrm{BC}$ group, $\mathrm{P}<0.01$; \# Compared with RYDS model group, $\mathrm{P}<$ 0.05 ; and \#\# compared with RYDS model group, $\mathrm{P}<0.01$. 


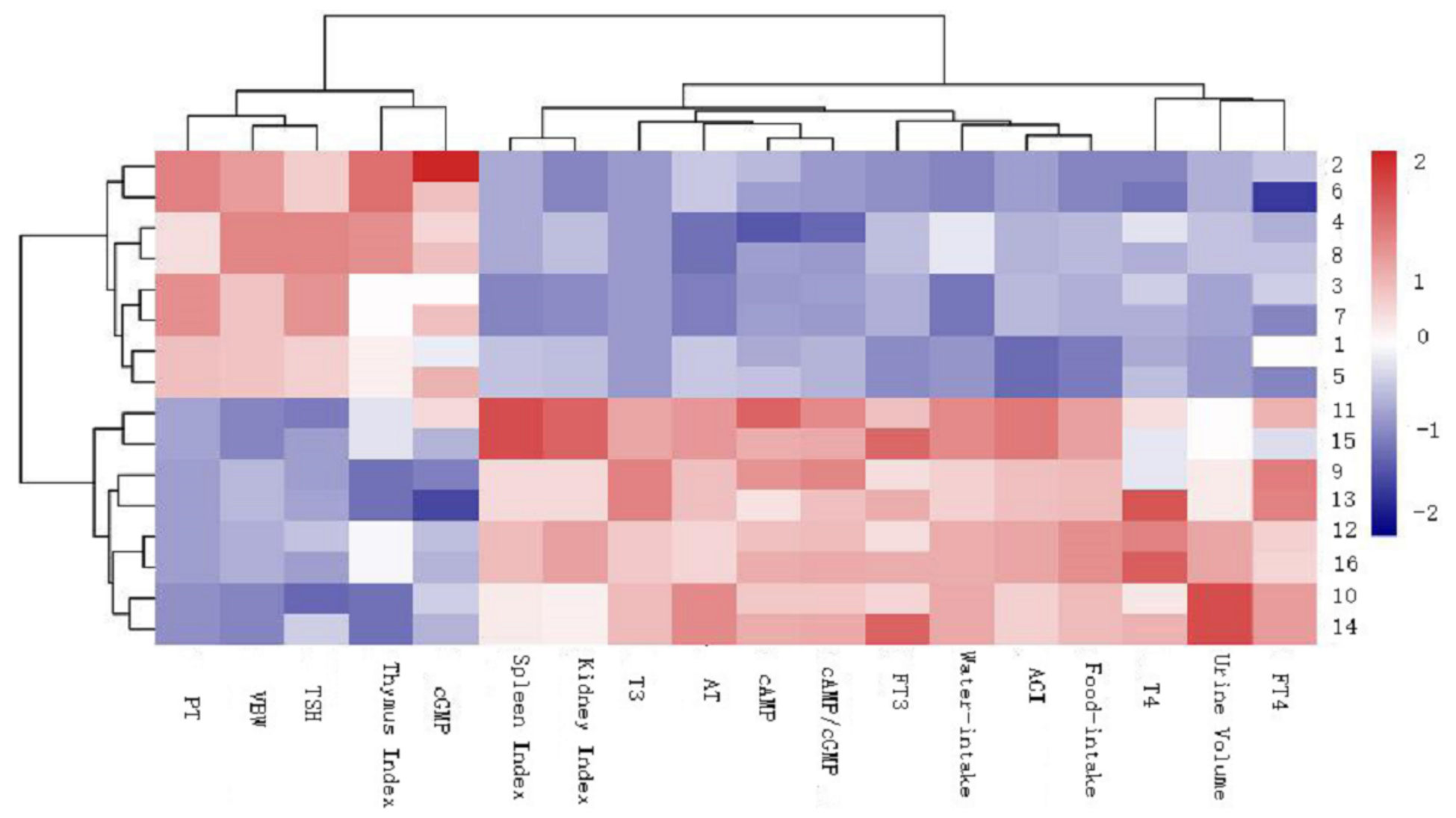

Figure 9

Heat Map of Cluster Analysis of the Values of the Detected Physiological and Endocrino-immunological Indexes of the Rats in the BC Group and RYDS Model Group. Notes: 1 - 8 = BC Group, 9 - 16 = RYDS Model Group, AGI = Adrenal Gland Index, AT = Anal Temperature, VBW = Variation in Body Weight, and PT $=$ Pain Threshold. 

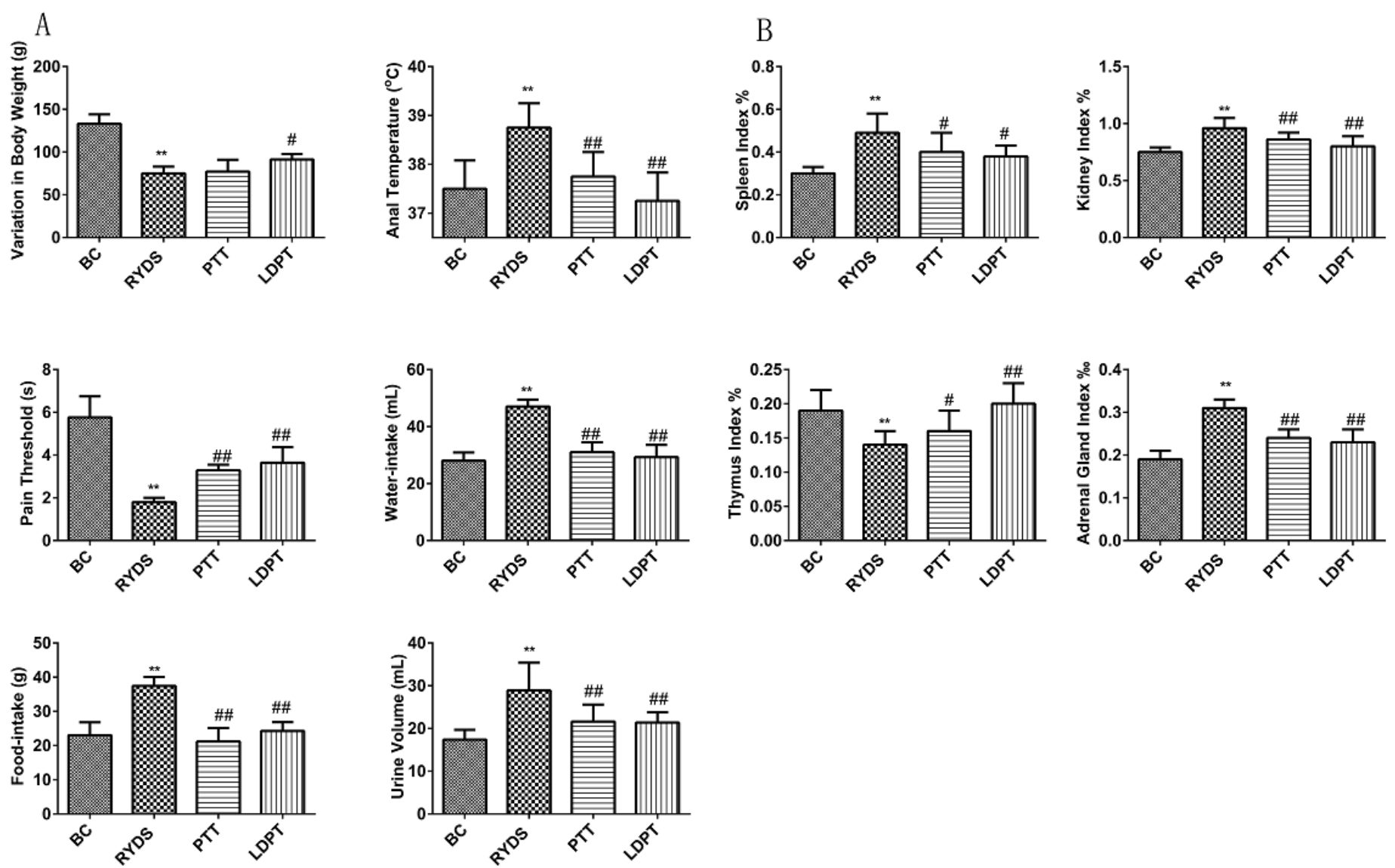

Figure 10

Effects of Raw AR, Salt Stir-fried AR and Mangiferin on Physiological Indexes and the Organ Indexes of Endocrine-immunological Organs of the Rats with RYDS. A = Data of the etection of physiological indexes. $\mathrm{B}=$ Data of the detection of the organs indexes of endocrine-immunological organs. All the data are expressed as the means $\pm S D(n=8)$. ${ }^{*}$ Compared with $B C$ group, $P<0.05$; ${ }^{* *}$ compared with $B C$ group, $\mathrm{P}<0.01$; \# Compared with RYDS model group, $\mathrm{P}<0.05$; and \#\# compared with RYDS model group, $\mathrm{P}<$ 0.01 . 

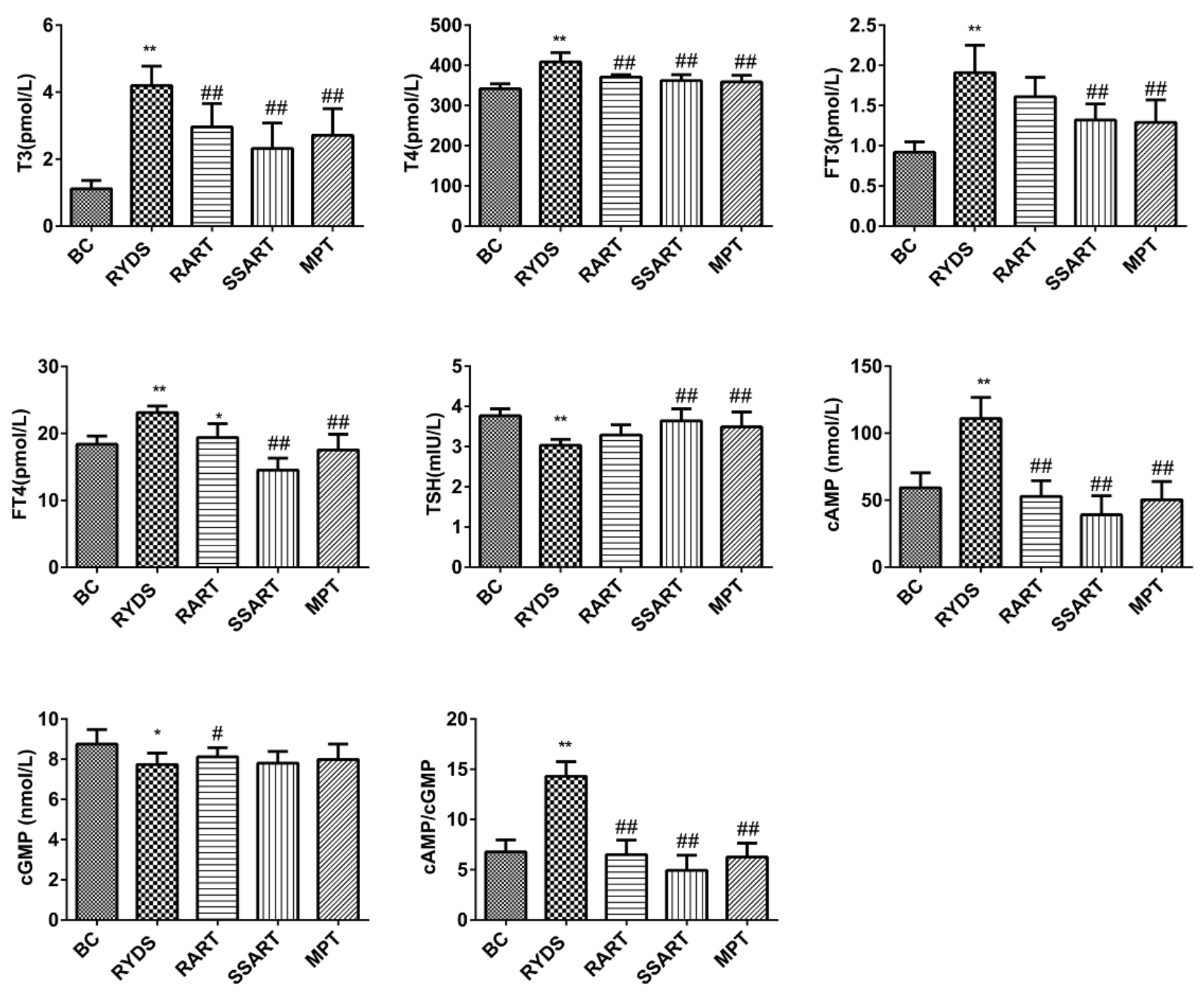

\section{Figure 11}

Effects of Raw AR, Salt Stir-fried AR and Mangiferin on Endocrine-immunological Indexes in Serum or Plasma of the Rats with RYDS. All the data are expressed as the means $\pm S D(n=8)$. ${ }^{*}$ Compared with BC group, $\mathrm{P}<0.05$; ${ }^{* *}$ compared with $\mathrm{BC}$ group, $\mathrm{P}<0.01$; \# Compared with RYDS model group, $\mathrm{P}<0.05$; and \#\# compared with RYDS model group, $\mathrm{P}<0.01$. 

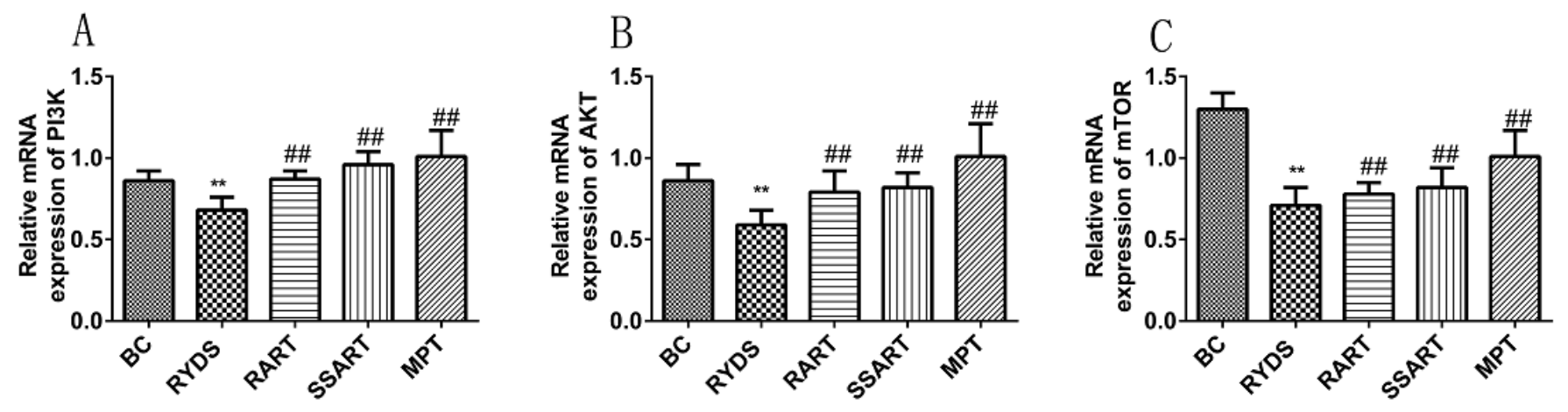

Figure 12

Effects of Raw AR, Salt Stir-fried AR and Mangiferin on mRNA Expression of PI3K/AKT/mTOR Pathway in Rats with RYDS. $A=$ The relative mRNA expression of PI3K. $B=$ The relative mRNA expression of AKT. $C=$ The relative mRNA expression of mTOR All the data are expressed as the means $\pm S D(n=8)$. *Compared with BC group, $\mathrm{P}<0.05$; ${ }^{*}$ compared with $\mathrm{BC}$ group, $\mathrm{P}<0.01$; \# Compared with RYDS model group, $\mathrm{P}<0.05$; and \#\# compared with RYDS model group, $\mathrm{P}<0.01$.
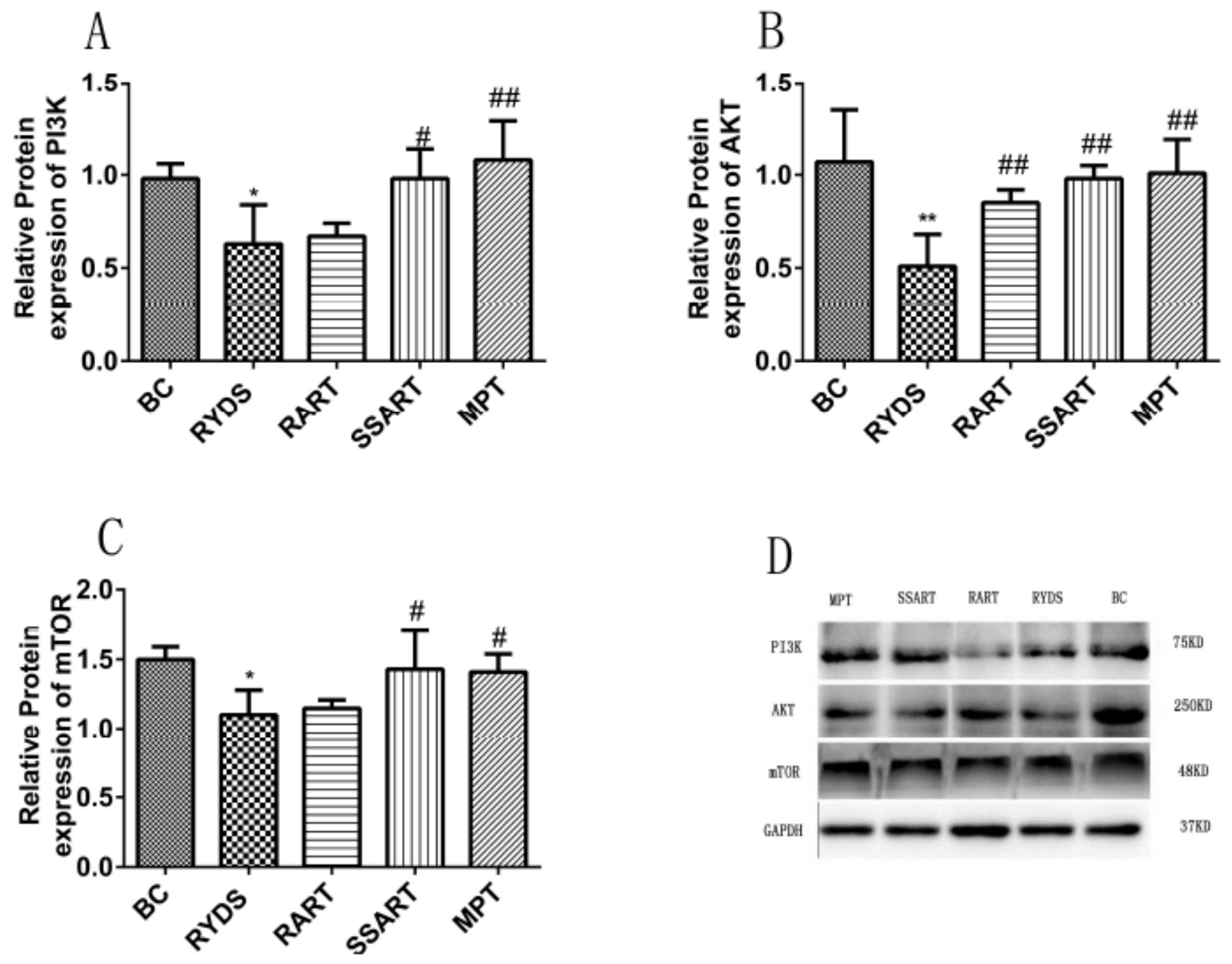
Figure 13

Effects of Raw AR, Salt Stir-fried AR and Mangiferin on Protein Expression of PI3K/AKT/mTOR Pathway in Rats with RYDS. $A=$ The relative protein expression of PI3K. $B=$ The relative protein expression of AKT. $\mathrm{C}=$ The relative protein expression of $\mathrm{mTOR} . \mathrm{D}=$ Representative western blot in $\mathrm{PI} 3 \mathrm{~K} / \mathrm{AKT} / \mathrm{mTOR}$ pathway.
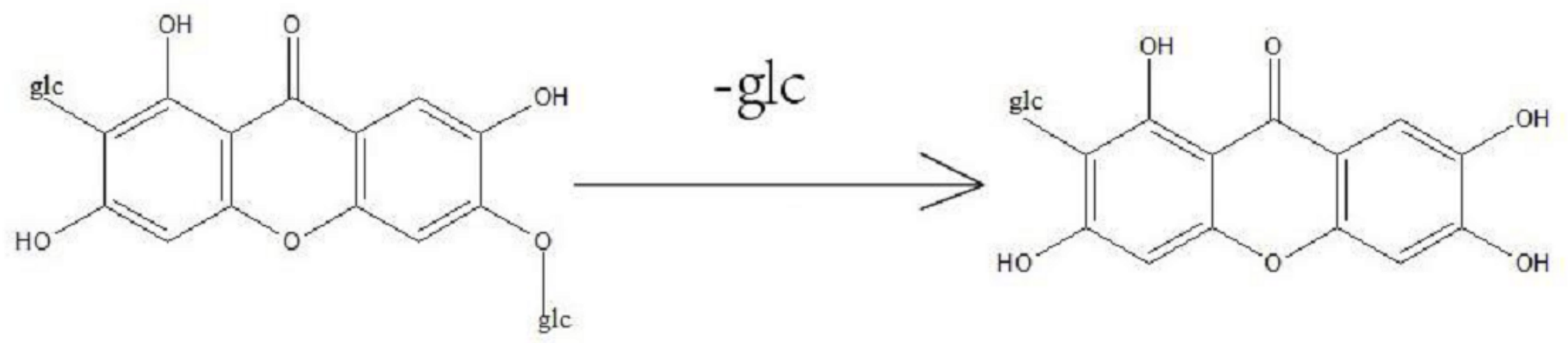

\section{Figure 14}

Possible Process of the Transformation of Neomangiferin to Mangiferin 